\title{
A New Transmissibility Based Indicator of Local Variation in Structure and Its Application for Damage Detection
}

\author{
X. Z. Li, Z. K. Peng, X. J. Dong, W. M. Zhang, and G. Meng \\ State Key Laboratory of Mechanical System and Vibration, Shanghai Jiao Tong University, Shanghai 200240, China \\ Correspondence should be addressed to Z. K. Peng; z.peng@sjtu.edu.cn
}

Received 4 May 2015; Accepted 24 June 2015

Academic Editor: Dong Wang

Copyright ( 2015 X. Z. Li et al. This is an open access article distributed under the Creative Commons Attribution License, which permits unrestricted use, distribution, and reproduction in any medium, provided the original work is properly cited.

\begin{abstract}
The presence of damage in engineering structures could usually cause local variation in stiffness or damping, and therefore it is meaningful to detect the variation as early as possible for protecting the engineering structure from serious damage. In the study, a novel method is developed to detect and locate the local variation in stiffness and damping for structures based on transmissibility. Some important properties of transmissibility are first analytically revealed and, further, a variation detection indicator is proposed to locate the variation. The effectiveness of the proposed method is verified by numerical studies and the usefulness of it is demonstrated by application for detecting crack position in beam structures. The results show that the proposed new method has better performance than the three conventional transmissibility based methods when considering different frequency bands and noise.
\end{abstract}

\section{Introduction}

Researchers have developed many useful vibration-based techniques for detecting structural damage, and enormous literatures [1-3] have been published to detect, locate, and quantify the damage. Normally, existence of structural damage in an engineering system leads to modification of the vibration. The damage caused by stiffness variation could alter the dynamic properties of the structure, such as natural frequencies and mode shapes. Therefore, feature extraction through vibration testing of healthy or damaged structures is the main core of vibration-based methods. One of the major challenges in structural health monitoring is the detection and location of small-scale damage since it is local damage. The difficulty mainly relies on two reasons: (i) the modal properties of the structure are also influenced by environmental parameters, such as load and boundary condition, which may cause changes in the vibration characters of the same order of magnitude as damage; (ii) modal parameters are global properties in nature and are only sensitive to substantial amounts of damage.

Transmissibility functions (TFs) [4], defined as the ratios of response in frequency domain or energy between two different DOFs of structure, have been a meaningful theory to evaluate the structural condition [5]. One key property of transmissibility functions is that it is local in nature. Zhang et al. [6] and Mottershead [7] demonstrated that transmissibility functions are determined solely by system zeros, which makes the transmissibility functions sensitive to the local variation. Structural damage affects both poles and zeros of system. While the poles are sensitive to the changes in structural health anywhere because it is a function of all the system parameters, the zeros are only sensitive to a certain subset of mass, damping, and stiffness that are localized in a region of the structure.

Taking advantage of this basic property, a large number of detection methods were proposed in the last 20 years [5]. In 1994, transmissibility functions were firstly proposed as potential features for damage detection by Chen et al. [8]. A neural network using TFs was developed to compute a novelty index for damage detection. After that, efforts have been made on the use of the transmissibility functions for local damage detection. In 1997, Schulz et al. [4] proposed a damage indicator using transmissibility of cross and autopower spectral density for the first time, which was used by many authors subsequently and was evolved into the spectra ratio of response directly later. The change of power transmissibility near damage was used to locate the crack. Then, Zhang et al. [6] used both magnitude and phase of transmissibility functions to improve the locating 


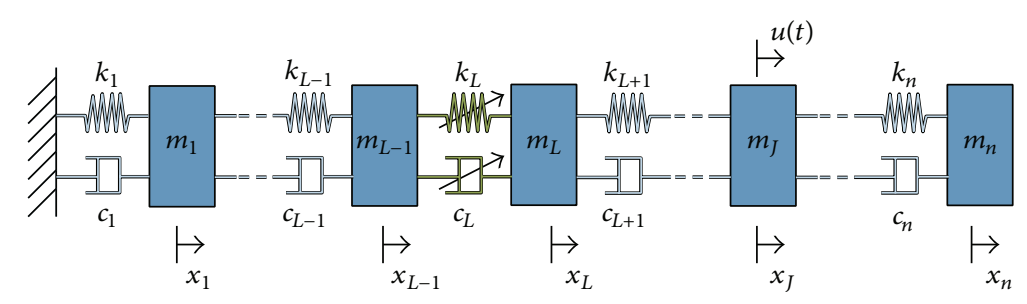

FIGURE 1: A one-dimensional MDOF system with local variation.

ability in 1999, and a concept of curvature TF was also developed to locate the damage. Experiment was made using cantilever beam to demonstrate the better ability than Schulz's method in the same frequency band. Johnson and Adams [9] extended the application of transmissibility to nonlinear system using quotient of transmissibility ratios in 2002. Structure with nonlinear damage can be also detected by the indicator in that literature.

Besides these methods proposed more than a decade ago, with the application of transmissibility in operational modal analysis $[10,11]$, some new indicators based on transmissibility were proposed for structural health monitoring in the last few years. In 2011, Maia et al. [12] extended the concept of Response Vector Assurance Criterion (RVAC) by replacing the FRF with transmissibility function in the indicator RVAC and damage quantification indicator (DRQ), which is the average value of RVAC along the frequency domain. The new method can detect and quantify the damage, but not location of damage. In 2013, Li and Hao [13] used the power spectral density transmissibility (PSDT) to detect the damage of shear connectors. PSDT matrices in the undamaged and damaged states or damaged states only were directly compared to identify the damage of shear connectors, while, in 2014, the same authors [14] proposed another method using transmissibility of frequency response function. By comparing the transmissibility vectors in undamaged and damaged states, the damage level of shear connectors can be identified. Then, in 2015, a method using response reconstruction with PSDT was published by them [15] again. In the same period, Zhou et al. proposed two new kinds of damage indicators using transmissibility in 2015. One kind of indicator [16] used the accumulation or modal assurance criterion of Transmissibility Coherence (TC) in frequency domain, which was defined as magnitude squared coherence. The detection results were compared with the FRF coherence and show a better performance. The other kind of damage detection approach [17] used the Mahalanobis distance of transmissibility as a damage feature. Mahalanobis distance can distinguish the damaged structural state condition from the undamaged one by considering the baseline data. While both kinds of method can quantify the damage severity, they cannot locate the damage.

The objective of the present research is to develop and test a novel method to detect local damage with transmissibility functions, which can be applied in linear and nonlinear MDOF systems. Properties of transmissibility functions in one-dimensional MDOF system with local variation are present, and a detection indicator is developed with the support of analytical derivation. Then, numerical simulations of linear, bilinear, and nonlinear systems are used to validate the properties and indicator. The technique is applied for crack position detection in beam structure and is compared with the three popular indicators by Schulz et al. [4], Zhang et al. [6], and Johnson and Adams [9].

\section{Transmissibility Functions of MDOF System}

2.1. MDOF System with Local Variation. Considering a general dynamic structural system with multiple-input and multiple-output, the motion equation of that system with local variation can be described as

$$
\mathbf{M} \ddot{\mathbf{x}}(t)+\mathbf{C} \dot{\mathbf{x}}(t)+\mathbf{K} \mathbf{x}(t)+\mathbf{F}_{\mathrm{LV}}(\dot{\mathbf{x}}(t), \mathbf{x}(t))=\mathbf{F}(t),
$$

where $\mathbf{M}, \mathbf{C}$, and $\mathbf{K}$ represent the $n \times n$ mass, damping, and stiffness matrices of the system, $\mathbf{x}(t)$ is the $n \times 1$ response vector, $\mathbf{F}(t)$ is the $n \times 1$ external force excitation, and $n \times 1$ vector $\mathbf{F}_{\mathrm{LV}}(\dot{\mathbf{x}}(t), \mathbf{x}(t))$ models the additional forces induced by the local variation.

For the sake of simplicity, consider the one-dimensional MDOF structure in Figure 1, which has only one local variation component and is subjected to one input excitation. Since TFs describe the connection between two different points of the system, it is more convenient to study their properties in one-dimensional system.

As shown in Figure 1, the Lth component has a local variation and a force excitation is acting on the $J$ th mass. The local variation induces the local restoring force $F_{v}$ between the $(L-1)$ th component and $L$ th component, which not only affect the element nearby but also change the dynamic properties of the whole system.

(i) If $F_{v}$ is a linear force, it can be described as

$$
F_{v}=k_{L}^{1} \Delta+c_{L}^{1} \dot{\Delta}
$$

where $k_{L}^{1}$ and $c_{L}^{1}$ are linear stiffness and damping coefficient and $\Delta=x_{L}-x_{L-1}, \dot{\Delta}=\dot{x}_{L}-\dot{x}_{L-1}$. Under this condition, the system is a linear system with linear local variation. And this kind of system might be caused by corrosion or loss in preload.

(ii) If $F_{v}$ is a bilinear force, it can be described as

$$
F_{v}= \begin{cases}k_{L}^{\prime} \Delta+c_{L}^{\prime} \dot{\Delta} & x_{L}(t)>0 \\ 0 & \text { else, }\end{cases}
$$

where $k_{L}^{\prime}$ and $c_{L}^{\prime}$ are the linear stiffness and damping coefficient which only connect with the $L$ th component. Under this condition, the system is a system with a bilinear component. This condition could be induced by the dry friction or damage of viscous-elastic material. 
(iii) If $F_{v}$ is a nonlinear force, according to Weierstrass Approximation Theorem [18], it is assumed that the restoring force can be described by the polynomial functions, yielding

$$
F_{v}=\sum_{i=1}^{P} k_{L}^{i} \Delta^{i}+\sum_{i=1}^{P} c_{L}^{i} \dot{\Delta}^{i}
$$

where $P$ is the polynomial degree and $k_{L}^{i}, c_{L}^{i}(i=2, \ldots, P)$ are nonlinear stiffness and damping coefficient. Under this condition, the system becomes a nonlinear system, which might be caused by the crack or loosened fasteners.

So, vector $\mathbf{F}_{\mathrm{LV}}$ in one-dimensional MDOF structure yields

$$
\mathbf{F}_{\mathrm{LV}}(t)=(\overbrace{0, \ldots, 0, F_{v}}^{L-2}-F_{v}, \overbrace{0, \ldots, 0}^{n-L})^{\prime} .
$$

And, the other parameters of this model are shown below. Consider that

$$
\begin{aligned}
\mathbf{M} & =\left[\begin{array}{cccc}
m_{1} & 0 & \cdots & 0 \\
0 & m_{2} & \cdots & 0 \\
\vdots & \vdots & \ddots & \vdots \\
0 & 0 & \cdots & m_{n}
\end{array}\right], \\
\mathbf{C} & =\left[\begin{array}{ccccc}
c_{1}+c_{2} & -c_{2} & 0 & \cdots & 0 \\
-c_{2} & c_{2}+c_{3} & -c_{3} & \ddots & \vdots \\
0 & \ddots & \ddots & \ddots & 0 \\
\vdots & \ddots & -c_{n-1} & c_{n-1}+c_{n} & -c_{n} \\
0 & \cdots & 0 & -c_{n} & c_{n}
\end{array}\right], \\
\mathbf{K} & =\left[\begin{array}{ccccc}
k_{1}+k_{2} & -k_{2} & 0 & \cdots & 0 \\
-k_{2} & k_{2}+k_{3} & -k_{3} & \ddots & \vdots \\
0 & \ddots & \ddots & \ddots & 0 \\
\vdots & \ddots & -k_{n-1} & k_{n-1}+k_{n} & -k_{n} \\
0 & \cdots & 0 & -k_{n} & k_{n}
\end{array}\right]
\end{aligned}
$$

$$
\begin{aligned}
& \mathbf{H}(s)=\left(\mathbf{M} s^{2}+\mathbf{C s}+\mathbf{K}\right)^{-1} \\
& =\left[\begin{array}{ccccc}
m_{1} s^{2}+\left(c_{1}+c_{2}\right) s+k_{1}+k_{2} & -c_{2} s-k_{2} & 0 & \cdots & 0 \\
-c_{2} s-k_{2} & m_{2} s^{2}+\left(c_{2}+c_{3}\right) s+k_{2}+k_{3} & -c_{3} s-k_{3} & \ddots & \vdots \\
0 & \ddots & \ddots & \ddots & 0 \\
\vdots & \ddots & -c_{n-1} s-k_{n-1} & m_{n-1} s^{2}+\left(c_{n-1}+c_{n}\right) s+k_{n-1}+k_{n} & -c_{n} s-k_{n} \\
0 & \cdots & 0 & -c_{n} s-k_{n} & m_{n} s^{2}+c_{n} s+k_{n}
\end{array}\right]^{-1}
\end{aligned}
$$

are the system mass, damping, and stiffness matrices, respectively. Consider that

$$
\begin{aligned}
\mathbf{x} & =\left(x_{1}, \ldots, x_{n}\right)^{\prime}, \\
\mathbf{F}(t) & =(\overbrace{0, \ldots, 0}^{J-1} u(t), \overbrace{0, \ldots, 0}^{n-J})^{\prime}
\end{aligned}
$$

are the displacement and external force vector, respectively. It is assumed that $J \geq L$.

Since this model could contain linear, bilinear, or nonlinear component, it will be used to find a suitable method to extend the concept of the traditional transmissibility into the nonlinear system directly and use the ratio of the frequency domain response to calculate TFs.

2.2. Transmissibility Functions and Properties of the System. A very powerful technique for solving the ordinary and partial differential equations describing the way in which certain quantities vary with time is that of the Laplace transform, which literally transforms these problems from time domain into an elementary algebraic expression [19]. Setting the initial conditions to zeros and applying the Laplace transform, the original motion equation yields

$$
\left(\mathbf{M} s^{2}+\mathbf{C} s+\mathbf{K}\right) \mathbf{x}(s)=\mathbf{F}_{\mathrm{LV}}(s)+\mathbf{F}(s),
$$

where $\mathbf{x}(s), \mathbf{F}(s)$ are the complex frequency domain response and excitation vectors, respectively, and $\mathbf{F}_{\mathrm{LV}}(s)$ is the complex frequency domain additional force induced by the local variation.

Therefore, the complex frequency domain response $\mathbf{x}(s)$ can be described as

$$
\mathbf{x}(s)=\mathbf{H}(s)\left(\mathbf{F}_{\mathrm{LV}}(s)+\mathbf{F}(s)\right),
$$

where detect the local variation in all kinds of structure. Here, we into complex frequency domain, from differential equations

is the transfer function matrix of the system. 
From the transfer function matrix, it can be known that the response is coupled, which means that the frequency response of one component is connected with that of the component before and after. And, according to the definition of TFs, the transmissibility of the MDOF system in the complex frequency domain can be derived from (9).

For any two consecutive components, the TFs can be expressed as

$$
T_{(i+1, i)}(s)=\frac{x_{i+1}(s)}{x_{i}(s)}
$$

where $i=1, \ldots, n-1$.

Thus, according to (11), when $i=1$, the first row of (9) yields

$$
\begin{gathered}
\left(m_{1} s^{2}+\left(c_{1}+c_{2}\right) s+\left(k_{1}+k_{2}\right)\right) x_{1}(s) \\
-\left(c_{2} s+k_{2}\right) x_{2}(s)=0 .
\end{gathered}
$$

So

$$
T_{(2,1)}(s)=\frac{m_{1} s^{2}+\left(c_{1}+c_{2}\right) s+\left(k_{1}+k_{2}\right)}{c_{2} s+k_{2}} .
$$

Subsequently, substituting (13) into the second row of (9) yields

$$
T_{(3,2)}(s)=\frac{m_{2} s^{2}+\left(1-1 / T_{(2,1)}\right)\left(c_{2} s+k_{2}\right)+c_{3} s+k_{3}}{c_{3} s+k_{3}} .
$$

Iteratively using the above procedure until $i=L-2$, it can be known that, for $2 \leq i \leq L-2$, the TFs yield

$$
\begin{aligned}
& T_{(i+1, i)}(s) \\
& \quad=\frac{m_{i} s^{2}+\left(1-1 / T_{(i, i-1)}\right)\left(c_{i} s+k_{i}\right)+c_{i+1} s+k_{i+1}}{c_{i+1} s+k_{i+1}} .
\end{aligned}
$$

Then, for $i=L-1$, substituting $T_{(L-1, L-2)}$ into the $(L-1)$ th row of (9) yields

$$
\begin{aligned}
& T_{(L, L-1)}(s) \\
& =\frac{m_{L-1} s^{2}+\left(1-1 / T_{(L-1, L-2)}\right)\left(c_{L-1} s+k_{L-1}\right)+c_{L} s+k_{L}}{c_{L} s+k_{L}}(1 \\
& \left.+\frac{F_{v}(s) / x_{L-1}(s)}{m_{L-1} s^{2}+\left(1-1 / T_{(L-2, L-1)}\right)\left(c_{L-1} s+k_{L-1}\right)+c_{L} s+k_{L}}\right) .
\end{aligned}
$$

And, for $i=L$

$$
\begin{aligned}
& T_{(L+1, L)}(s) \\
& =\frac{m_{L} s^{2}+\left(1-1 / T_{(L, L-1)}\right)\left(c_{L} s+k_{L}\right)+c_{L+1} s+k_{L+1}}{c_{L+1} s+k_{L+1}}(1 \\
& \left.-\frac{F_{v}(s) / x_{L}(s)}{m_{L} s^{2}+\left(1-1 / T_{(L, L-1)}\right)\left(c_{L} s+k_{L}\right)+c_{L+1} s+k_{L+1}}\right) .
\end{aligned}
$$

Since $T_{(i+1, i)}$ is complicated for $i=L-1, L$, it is difficult to calculate and judge the TFs for the next components. However, a similar procedure can be conducted starting from the $n$th row of (9). So, for $i=n$

$$
T_{(n, n-1)}(s)=\frac{c_{n} s+k_{n}}{m_{n} s^{2}+c_{n} s+k_{n}} .
$$

Subsequently, for $J+1 \leq i \leq n-1$

$$
\begin{aligned}
& T_{(i, i-1)}(s) \\
& \quad=\frac{c_{i} s+k_{i}}{m_{i} s^{2}+\left(1-T_{(i+1, i)}\right)\left(c_{i+1} s+k_{i+1}\right)+c_{i} s+k_{i}} .
\end{aligned}
$$

Then, when $i=J$

$$
\begin{aligned}
& T_{(J, J-1)}(s) \\
& \quad=\frac{c_{J} s+k_{J}}{m_{J} s^{2}+\left(1-T_{(J+1, J)}\right)\left(c_{J+1} s+k_{J+1}\right)+c_{J} s+k_{J}}(1 \\
& \left.\quad+\frac{u(s) / x_{J-1}(s)}{c_{J} s+k_{J}}\right) .
\end{aligned}
$$

Until now, the TFs in complex frequency domain have been calculated for this MDOF system. Then, we need to transfer these functions into frequency domain. Since the Fourier transform is a special case of the Laplace transform [19], the frequency domain of TFs yields

$$
T_{(i+1, i)}(j \omega)=\left.T_{(i+1, i)}(s)\right|_{s=j \omega} .
$$

The equality holds up if and only if the convergence region of the Laplace transform contains the imaginary axis. After TFs in frequency domain are got from (21), the influence of the local variation could be studied by sensitivity.

According to the TFs above, the whole system is divided into four kinds of components.

(i) For $1 \leq i \leq L-2$ and $J \leq i \leq n-1$

$$
\begin{aligned}
& \frac{\partial T_{(i+1, i)}(j \omega)}{\partial F_{v}(j \omega)}=0, \\
& \frac{\partial T_{(i+1, i)}(j \omega)}{\partial u(j \omega)}=0 .
\end{aligned}
$$

(ii) For $i=L-1, L$

$$
\begin{aligned}
& \frac{\partial T_{(i+1, i)}(j \omega)}{\partial F_{v}(j \omega)} \neq 0, \\
& \frac{\partial T_{(i+1, i)}(j \omega)}{\partial u(j \omega)}=0 .
\end{aligned}
$$

(iii) For $i=J-1$

$$
\begin{aligned}
& \frac{\partial T_{(i+1, i)}(j \omega)}{\partial F_{v}(j \omega)}=0, \\
& \frac{\partial T_{(i+1, i)}(j \omega)}{\partial u(j \omega)} \neq 0 .
\end{aligned}
$$


(iv) For $L<i<J-1$

$$
\begin{aligned}
& \frac{\partial T_{(i+1, i)}(j \omega)}{\partial F_{v}(j \omega)} \neq 0, \\
& \frac{\partial T_{(i+1, i)}(j \omega)}{\partial u(j \omega)} \neq 0 .
\end{aligned}
$$

It is difficult to calculate the sensitivity of TFs to $F_{v}(j \omega)$, $u(j \omega)$ for $L<i<J-1$ directly. But, from the derivation of the TFs at $i=L-1, L, J$, it can be known that transmissibility should be influenced by $F_{v}(j \omega)$ and $u(j \omega)$.

One conclusion can be got from the sensitivity study directly, which is that the TFs are influenced by the local variation as well as the location of excitation. This conclusion agrees with the research before [9]. With further study, the TFs in case (i) are irrelevant to the stiffness and damping coefficients $k_{L}^{i}, c_{L}^{i}$ and excitation $u(j \omega)$. For instance, the transmissibility function at $i=1$ is always equal to $\left(-m_{1} \omega^{2}+\right.$ $\left.\left(c_{1}+c_{2}\right) j \omega+\left(k_{1}+k_{2}\right)\right) /\left(c_{2} j \omega+k_{2}\right)$, no matter that $k_{L}^{i}$ is nonzero or not. While the TFs in cases (ii) and (iii) are influenced directly by the local variation and excitation, case (iv) is affected mediately.

For $i=L-1$, if there is no local variation, TFs yield

$$
\begin{aligned}
& \bar{T}_{(L, L-1)}(j \omega) \\
& =\frac{-m_{L-1} \omega^{2}+\left(1-1 / T_{(L-1, L-2)}\right)\left(c_{L-1} j \omega+k_{L-1}\right)+c_{L} j \omega+k_{L}}{c_{L} j \omega+k_{L}} .
\end{aligned}
$$

Set $\alpha=\left(F_{v}(j \omega) / x_{L-1}(j \omega)\right) /\left(-m_{L-1} \omega^{2}+(1-1 /\right.$ $\left.\left.T_{(L-2, L-1)}\right)\left(c_{L-1} j \omega+k_{L-1}\right)+c_{L} j \omega+k_{L}\right)$, and then

$$
\frac{T_{(L, L-1)}(j \omega)}{\bar{T}_{(L, L-1)}(j \omega)}=(1+\alpha) .
$$

So, (27) shows the difference of the TFs before and after the local variation. The difference grows with the increase of $\alpha$. And $\alpha$ changes with different frequency. When frequency is near the natural frequency, $x_{L-1}(j \omega)$ will be much bigger than that of the frequency far away from the natural frequency. If the local variation and excitation force are constant, then $\alpha$ near the natural frequency will be smaller than that of the other frequency bands. That means the difference between the TFs before and after the local variation will be smaller than that of the other frequency bands.

While for $i=J$, if there is no excitation, TFs yield

$$
\begin{gathered}
\bar{T}_{(J, J-1)}(j \omega) \\
=\frac{c_{J} j \omega+k_{J}}{-m_{J} \omega^{2}+\left(1-T_{(J+1, J)}\right)\left(c_{n+1} j \omega+k_{n+1}\right)+c_{J} j \omega+k_{J}} . \\
\text { Set } \beta=\left(u(j \omega) / x_{J-1}(j \omega)\right) /\left(c_{J} j \omega+k_{J}\right), \text { and then } \\
\frac{T_{(J, J-1)}(j \omega)}{\bar{T}_{(J, J-1)}(j \omega)}=(1+\beta) .
\end{gathered}
$$

Similar to (27), (29) shows the influence of the external excitation to the transmissibility. If $u(j \omega)$ is constant, $\beta$ becomes small when the frequency is near the natural frequency. That means the influence of the external excitation is much weak in the frequency bands near the natural frequency.

Thus, from the discussion above, two properties of TFs of the MDOF structure can be got.

Property 1. In theory, $T_{(i+1, i)}(j \omega)$ is irrelevant to the stiffness and damping coefficients $k_{L}^{i}, c_{L}^{i}(i=1, \ldots, P)$ and external excitation $u(j \omega)$ for components in the range of $1 \leq i \leq L-2$ and $J \leq i \leq n-1$, while, for $L-1 \leq i \leq J-1, T_{(i+1, i)}(j \omega)$ is influenced by these parameters.

Property 2. To increase the sensitivity of TFs to the local variation, frequency bands should be selected narrowing the range of natural frequency, while, to decrease the dependence of TFs on external excitation, frequency bands should include the range of natural frequency.

Property 1 can be used to detect the local variation by TFs, because it gives the discrimination between the positions with and without local variation. And Property 2 validates the conclusion again that it is difficult to decide the frequency bands of the TFs until the test date got from the practice.

2.3. Detection Indicator Based on Transmissibility. From the two properties, it is revealed that the difference between the transmissibilities $T_{(i+1, i)}(j \omega)$ and $\bar{T}_{(i+1, i)}(j \omega)$ is zero for the interval without the restoring force and external excitation in theory, while, for the other components, it is nonzero. Therefore, an indicator can be developed to detect the local variation using the transmissibility difference between the intact structure and local variation structure:

$$
\begin{array}{r}
\mathrm{TD}_{i}=\sum_{\omega_{0}}^{\omega_{N}} w_{i}(j \omega)\left|T_{(i+1, i)}(j \omega)-\bar{T}_{(i+1, i)}(j \omega)\right| \\
(i=1, \ldots, n-1),
\end{array}
$$

where $\omega \in\left(\omega_{0}, \omega_{N}\right)$ is the frequency band and

$$
\begin{aligned}
& w_{i}(j \omega) \\
& \quad=\frac{x_{i+1}(j \omega)+x_{i}(j \omega)+\bar{x}_{i+1}(j \omega)+\bar{x}_{i}(j \omega)}{\sum_{\omega_{0}}^{\omega_{N}}\left(x_{i+1}(j \omega)+x_{i}(j \omega)+\bar{x}_{i+1}(j \omega)+\bar{x}_{i}(j \omega)\right)}
\end{aligned}
$$

is the factor, which is used to increase the weight of resonance. According to the two properties, frequency band including the resonance could reduce the influence of excitation. Hence, in order to increase the sensitivity of the indicators to damage, not the excitation, the weighting factor is added to the differences of TFs before and after local variation. This indicator is a linear sum along all the spectral lines of the functions, and the frequency band can be chosen differently for each case.

\section{Simulation Studies on Different Kinds of MDOF Systems}

Since the restoring force $F_{v}$ could be linear, bilinear, or nonlinear, the indicator should be useful in all kinds of system. In 
TABLE 1: Comparison of the natural frequencies before and after variation.

\begin{tabular}{lcccccccc}
\hline Modes & 1 & 2 & 3 & 4 & 5 & 6 & 7 & 8 \\
\hline $\bar{\omega}_{n}$ & 5.536 & 16.420 & 26.744 & 36.158 & 44.341 & 51.013 & 55.948 & 58.978 \\
$\omega_{n}$ & 5.276 & 16.251 & 24.903 & 36.066 & 41.918 & 49.793 & 54.993 & 57.502 \\
$\left|\omega_{n}-\bar{\omega}_{n}\right| / \bar{\omega}_{n}(\%)$ & 4.697 & 1.029 & 6.884 & 0.254 & 5.464 & 2.392 & 1.707 & 2.503 \\
\hline
\end{tabular}

this section, numerical simulation will be studied on MDOF systems with linear, bilinear, or nonlinear component to validate the properties and detection indicator developed in the previous section.

3.1. Detection Indicators of System with Linear Local Variation. A linear local variation system is the case when the initially linear-elastic structure remains linear-elastic after local variation. The changes in dynamic properties could be induced by changes in the geometry or the material properties of the structure, but the structural response can still be modeled using linear equations of motion. Lots of methods have been proposed to detect and identify the local variation or damage in such system. For instance, in [20], a nondestructive method using measurements of the structural natural frequency was described to detect, locate, and quantify damage of an aluminum plate. And change in the flexibility matrix was used to detect and locate damage on a wide-flange steel beam in [21], while, in this simulation, the ability of detection indicator proposed in Section 2 will be demonstrated in linear system.

A linear local variation system with 8 degrees of freedom is built using the one-dimensional MDOF model in Figure 1. The linear variation locates in the 4 th component, so $L=4$. An impulse excitation is applied on the 6th mass, so $J=6$. It is assumed that the damping is proportional to the stiffness; for example, $\mathbf{C}=\mu \mathbf{K}$. The values of the system parameters are taken as

$$
\begin{aligned}
m_{1} & =\cdots=m_{8}=1, \\
k_{1} & =\cdots=k_{8}=3.5531 \times 10^{4}, \\
\mu & =0.001, \\
u(t) & = \begin{cases}2 & t=0 \\
0 & t \neq 0,\end{cases} \\
k_{L}^{1} & =-0.4 k_{4} .
\end{aligned}
$$

The fourth-order Runge-Kutta method is used to calculate the system responses. And the spectra of the responses can be got through the FFT. Then, TFs can be determined using the frequency responses.

Table 1 shows the first 8 natural frequencies of the system before and after the variation, which indicates that the small local variation is difficult to detect from the change of natural frequencies. The frequency band of the transmissibility is determined by including the first 5 natural frequencies and is set as $0-50 \mathrm{~Hz}$.
The TFs of the 8 points are shown in Figure 2. And their differences in frequency band of $0-50 \mathrm{~Hz}$ are also calculated. For $i=1,2, T_{(2,1)}, T_{(3,2)}$ are very close to $\bar{T}_{(2,1)}, \bar{T}_{(3,2)}$, respectively, except for few frequency points near the 6th natural frequency. And $T_{(6,7)}, T_{(7,8)}$ and $\bar{T}_{(6,7)}, \bar{T}_{(7,8)}$ are similar to them, while, for $i \stackrel{=}{=} 3,4,5$, there are much bigger differences between the TFs before and after the variation. Thus, the results agree with the properties in the last section that TFs are influenced by the local variation and excitation. In addition, the differences are not always very big in the whole frequency band even for the TFs influenced by the local variation and input directly. That means not all the frequencies are sensitive to this kind of local variation. On the other hand, TFs away from the local variation and input are not all exactly equal to zero. That might be caused by the error from the resonance and antiresonance.

Then, indicators of this linear system are calculated and the results are shown in Figure 3. In this system, stiffness and damping variation locate in the 4th component, which connect the 3rd and 4th component. And $\mathrm{TD}_{3}$, the transmissibility indicator between the 3rd and 4th components, is the maximum of all the indicators. So, the position of the local variation is detected by the indicators correctly. Since the external force is applied on the 6th component, the 3rd, 4th, and 5th indicators are bigger than the others. So, this is consistent with Property 1 that the transmissibility between the local variation and external force is influenced, while others are not. So, for this system, local variation is indicated correctly.

\subsection{Detection Indicators of System with Bilinear Component.} A local bilinear variation system is the case when the initially linear-elastic structure changes to nonlinear structure because of bilinear component. One example of this kind of system is the formation of a fatigue crack that subsequently opens and closes under the normal operating vibration environment. Rivola and White [22] provided a possibility of using the bispectral analysis technique to detect damage in structures and conducted simulation and experiment test on a beam with a fatigue crack. In [23], the authors found that the numerically generated steady state response of the system betrays the presence of the bilinear spring. So, this phenomenon was used to determine crack location, depth, and opening load in a cracked beam. In [24], a solution based on the use of two square wave functions to model stiffness change was proposed for bilinear oscillators under low-frequency excitation, and the predicted spectral pattern and the magnitude of each harmonic component for a simply supported beam showed the existence of crack. Since 

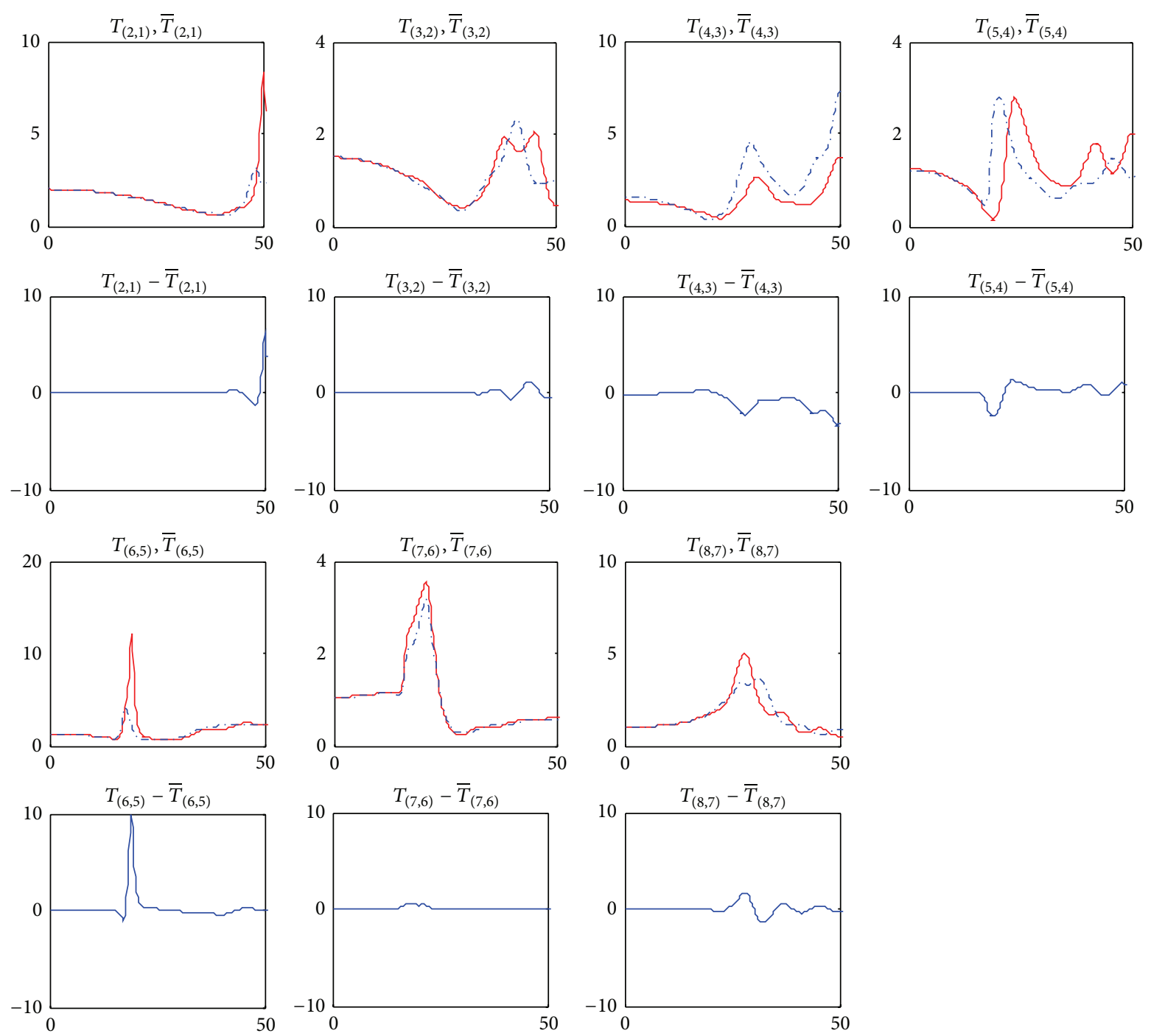

FIGURE 2: TFs of the system with linear local variation before and after the variation and their difference versus frequency $(\mathrm{Hz})$ : blue $-T_{(i+1, i)}$, red- $\bar{T}_{(i+1, i)}$.

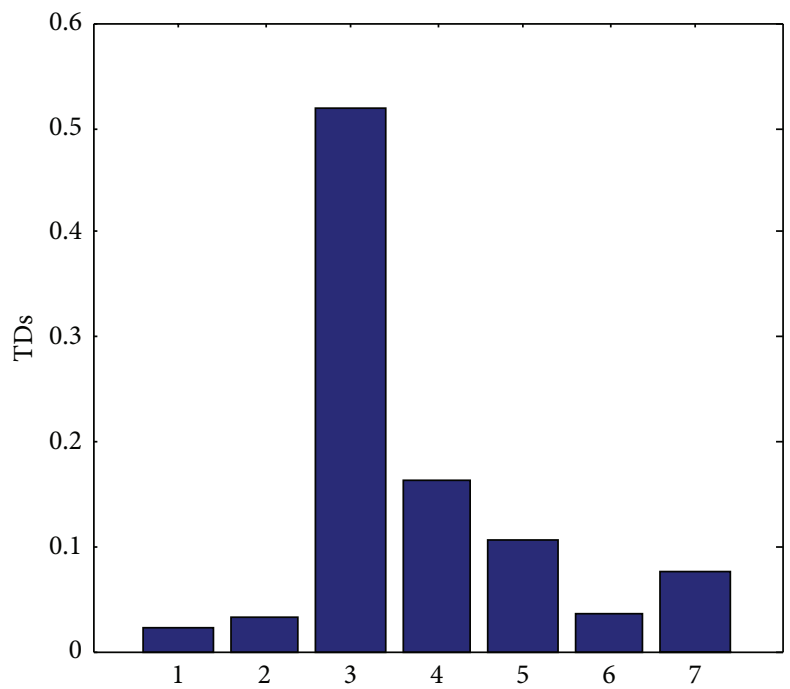

FIGURE 3: Detection indicators of the system with linear local variation. 

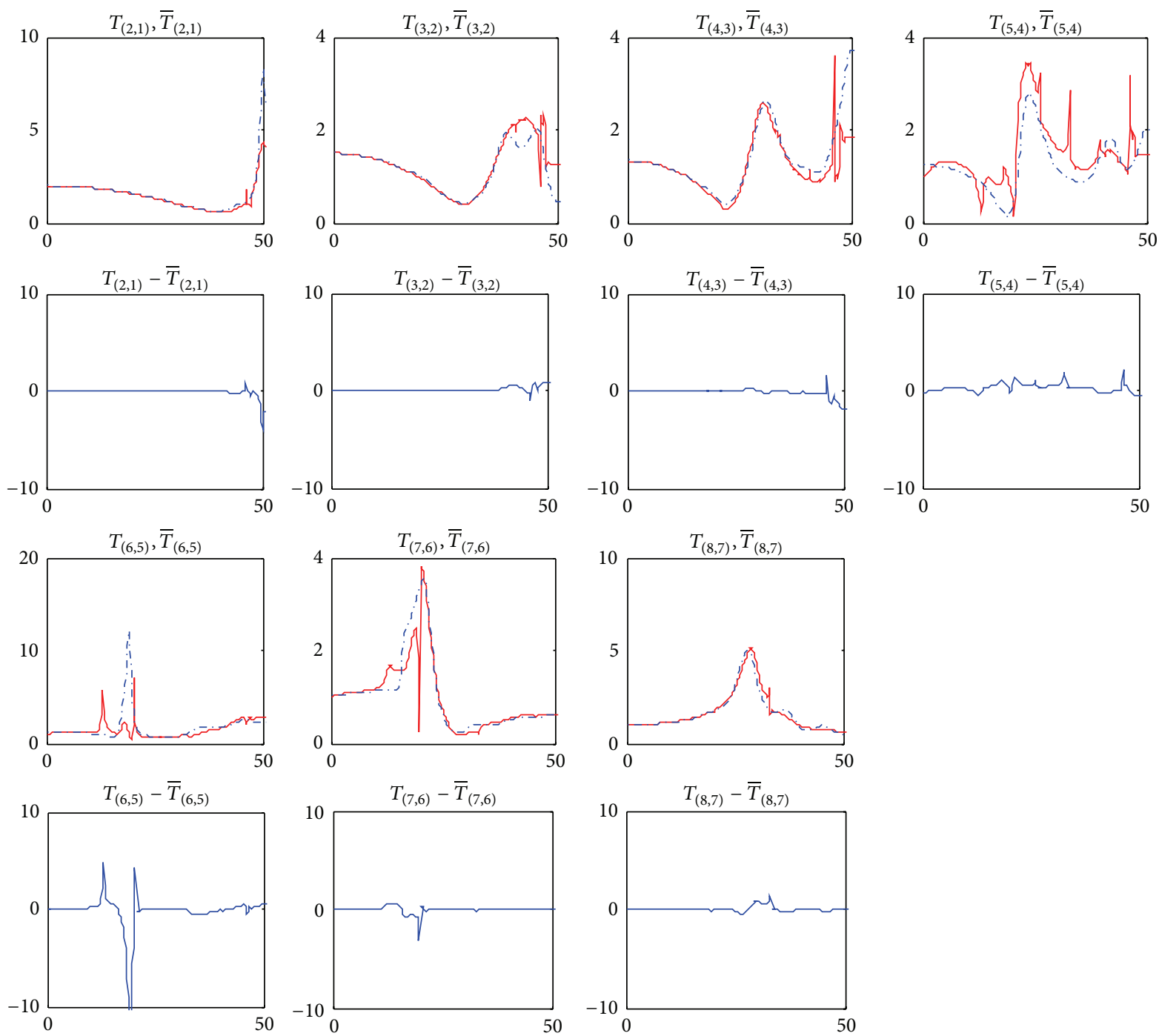

FIGURE 4: TFs of the system with bilinear component before and after the variation and their difference versus frequency $(\mathrm{Hz})$ : blue- $T_{(i+1, i)}$, red- $\bar{T}_{(i+1, i)}$.

the bilinear system is a useful system in the crack detection, it will be used to validate the indicator of TFs here.

In this numerical simulation, the 4 th component of one 8 -DOF system contains bilinear stiffness; that is, $L=4$. And an impulse excitation is applied on the 6th mass; that is, $J=6$. The values of the system parameters are the same as the linear system in Section 3, except the additional stiffness $k^{\prime}$ attached to the 4 th component alone. The value of $k^{\prime}$ is taken as

$$
k^{\prime}= \begin{cases}0.6 k_{4} & x_{4}(t)>0 \\ 0 & \text { else }\end{cases}
$$

where $k_{4}$ and $x_{4}(t)$ are linear stiffness and time response of the 4 th component, respectively.

Then, the responses are calculated by the fourth-order Runge-Kutta method and response spectrums can be got.
Figure 4 shows the TFs of the system with bilinear component. It can be seen that, for $i=1,2,3,6,7, T_{(i+1, i)}$ are close to $\bar{T}_{(i+1, i)}$, while, for $i=4,5$, there is much difference between them. Since the bilinear variation locates in the 4 th component solely, $T_{(5,4)}$ is affected seriously. And $T_{(6,5)}$ is also influenced by location of the input force.

Then, detection indicators are calculated by the response spectrum of the 8 points. In Figure $5, \mathrm{TD}_{4}$ and $\mathrm{TD}_{5}$ are much bigger than the other indicators. And $\mathrm{TD}_{4}$ is a little bigger than $\mathrm{TD}_{5}$, so the local bilinear variation can be detected. In this simulation, it can be seen that the influence of the input force is strong compared with the local variation. So, location of the input force should be determined carefully.

3.3. Detection Indicators of System with Nonlinear Component. A nonlinear local variation system is the case when 


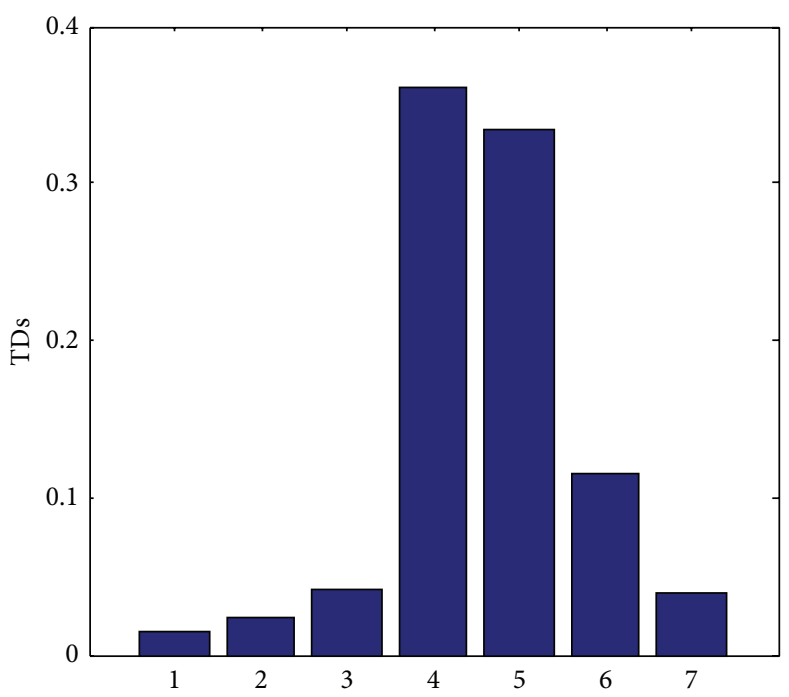

Figure 5: Detection indicators of the system with bilinear component.

the initially linear-elastic structure behaves in a nonlinear manner after the local nonlinear variation has been introduced. Its examples include loose connection, crack, and nonlinear material. Since the nonlinear variation is much complicated, some new nondestructive methods were proposed in the last few years. Ultrasonic wave is one of these methods. In [25], nonlinear Rayleigh surface wave was used to evaluate the fatigue damage induced by plastic deformation in A36 steel. And, in [26-28], another kind of ultrasonic wave, nonlinear Lamb wave, was used to detect and locate the nonlinear damage caused by material nonlinearity. The method even predicted the remaining lifetime of the structures. These two methods of the ultrasonic wave are normally based on the fact that the nonlinear wave could be induced by the damage. Compared with the traditional methods, these methods are much more sensitive to the damage, but strict requirements for the experiment equipment are needed. In this section, simulation will show the application of TFs to the nonlinear damage. This method is much more convenient compared to ultrasonic wave methods and is more sensitive to nonlinear damage compared with the traditional methods.

A nonlinear system is developed in this section. The nonlinear variation locates in the 4 th component and we apply the impulse force on the 6th mass. So, $L=4$ and $J=6$. The nonlinear stiffness and damping are set as

$$
\begin{aligned}
k_{L}^{2} & =0.4 k_{4}^{2}, \\
k_{L}^{3} & =1.4 k_{4}^{3}, \\
c_{L}^{2} & =0.001 \times 0.4 k_{4}^{2}, \\
c_{L}^{3} & =0.001 \times 1.4 k_{4}^{3} .
\end{aligned}
$$

The other parameters of the nonlinear system are taken as the same values as the linear system. Then, the same procedure is applied on this nonlinear system and the results are shown in Figures 6 and 7.
In Figure 6, the results show great accordance to the properties proposed in Section 2. The local variation and position of input influence the transmissibility nearby. Then, indicators are calculated in Figure 7. The maximum value $\mathrm{TD}_{3}$ shows the position of the nonlinear variation.

Through the three simulation cases in this section, properties of the transmissibility functions can be validated and the indicator shows a great performance in the local variation detection in linear, bilinear, and nonlinear systems. It also can be seen that location of external force has a strong influence on the value of the indicator.

\section{Application for Crack Position Detection in Beam Structures}

This section is focused on the crack position detection in cantilever beam. The responses of the beam structures with and without damage are calculated using the finite element model. So, the detection indicators can be got from their differences.

4.1. Model Description. The local variation is implemented using open crack. There are a number of methods to model crack in structures [29-33]. A simplified model of open crack by Sinha et al. [34] is adopted in this paper. In this model, for the area close to the crack, the stiffness is given by

$$
\begin{aligned}
& E I(\xi) \\
& = \begin{cases}E I_{0}-E\left(I_{0}-I_{c j}\right) \frac{\left(\xi-\xi_{j 1}\right)}{\left(\xi_{j}-\xi_{j 1}\right)} & \xi_{j 1} \leq \xi \leq \xi_{j} \\
E I_{0}-E\left(I_{0}-I_{c j}\right) \frac{\left(\xi_{j 2}-\xi\right)}{\left(\xi_{j 2}-\xi_{j}\right)} & \xi_{j} \leq \xi \leq \xi_{j 2},\end{cases}
\end{aligned}
$$



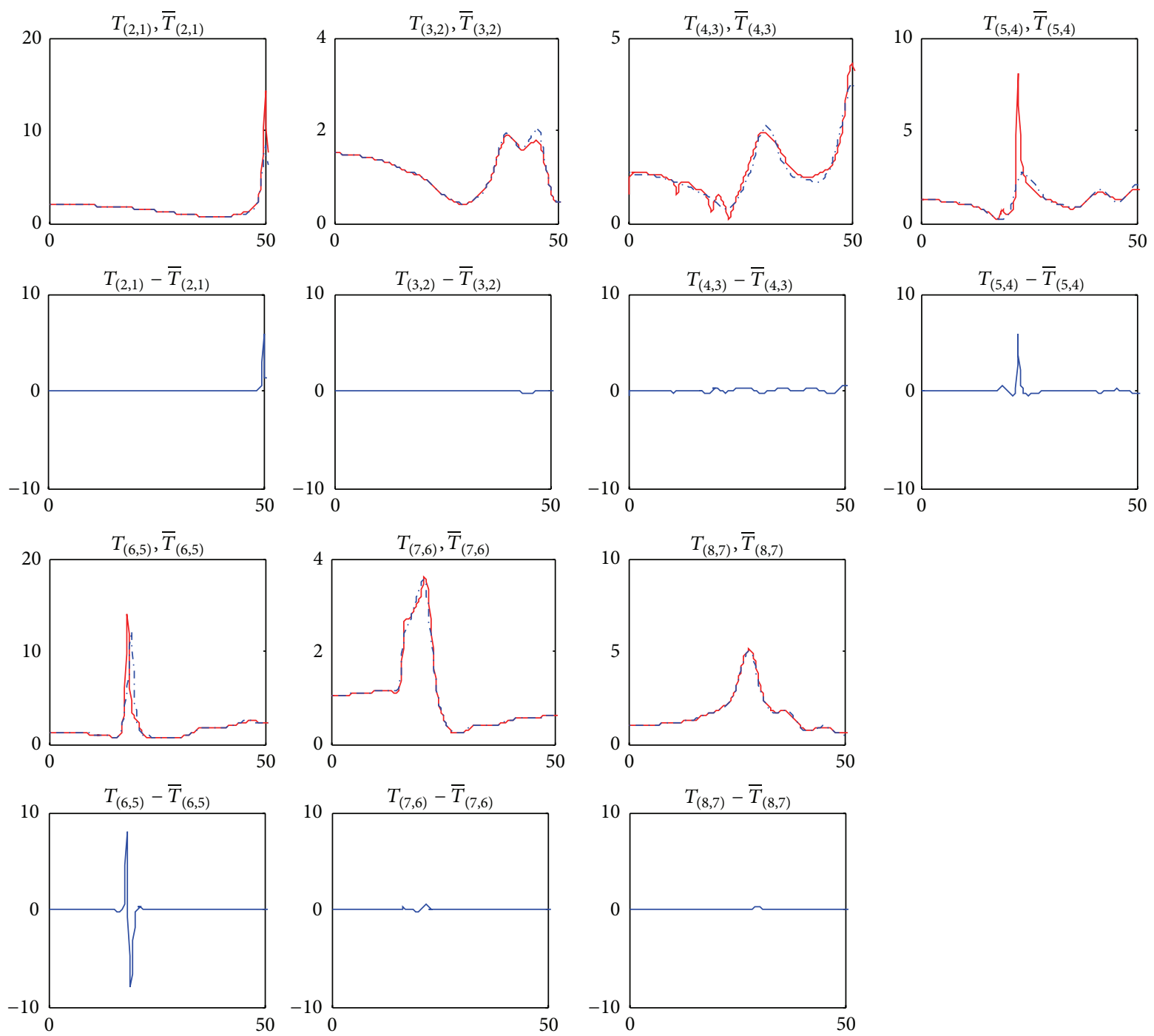

FIGURE 6: TFs of the system with nonlinear component before and after the variation and their difference versus frequency $(\mathrm{Hz})$ : blue- $T_{(i+1, i)}$, red- $\bar{T}_{(i+1, i)}$.

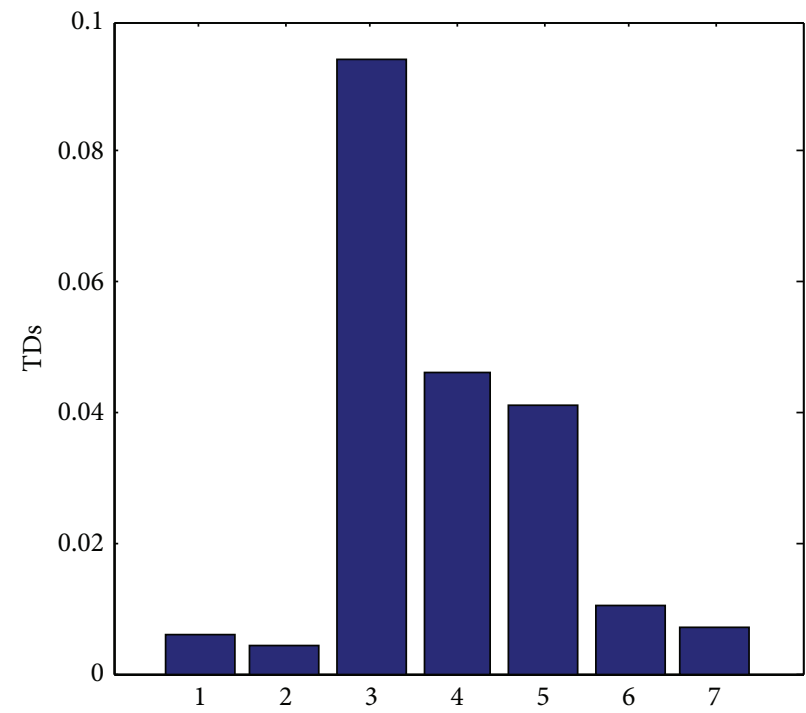

FIGURE 7: Detection indicators of the system with nonlinear component. 


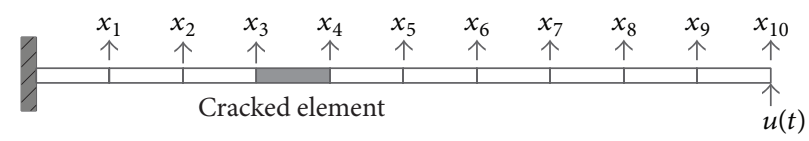

FIGURE 8: Finite element model of a cantilever beam.

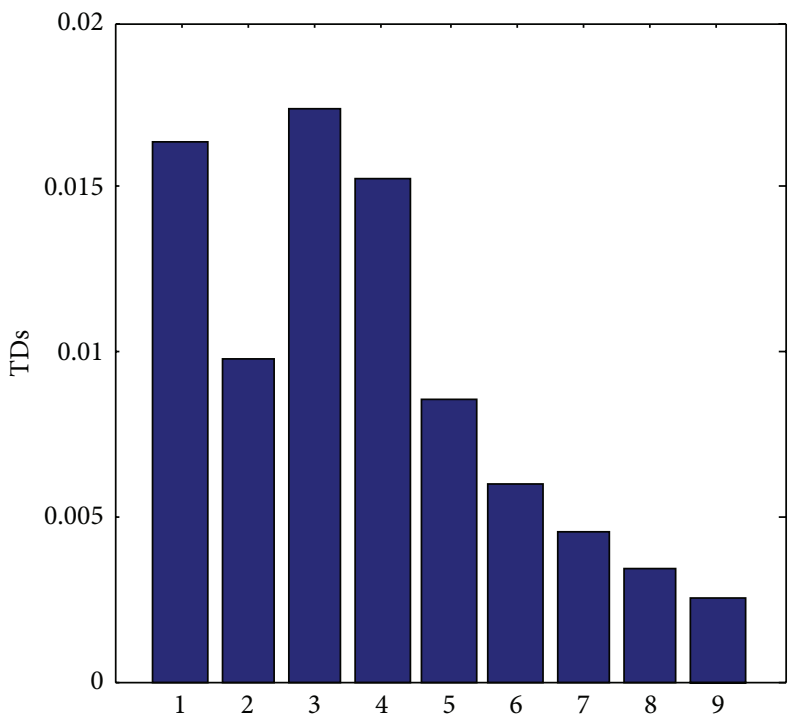

(a)

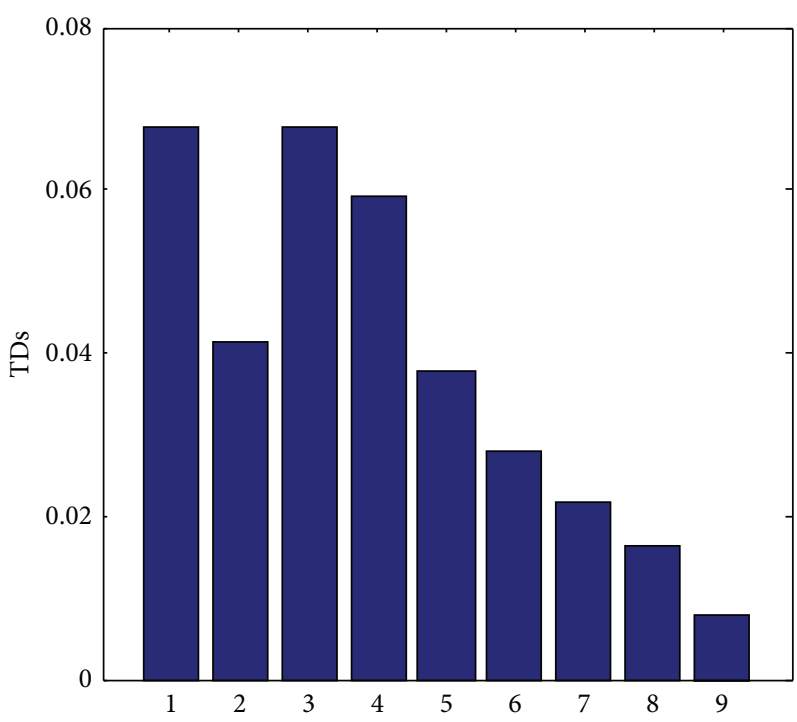

(c)

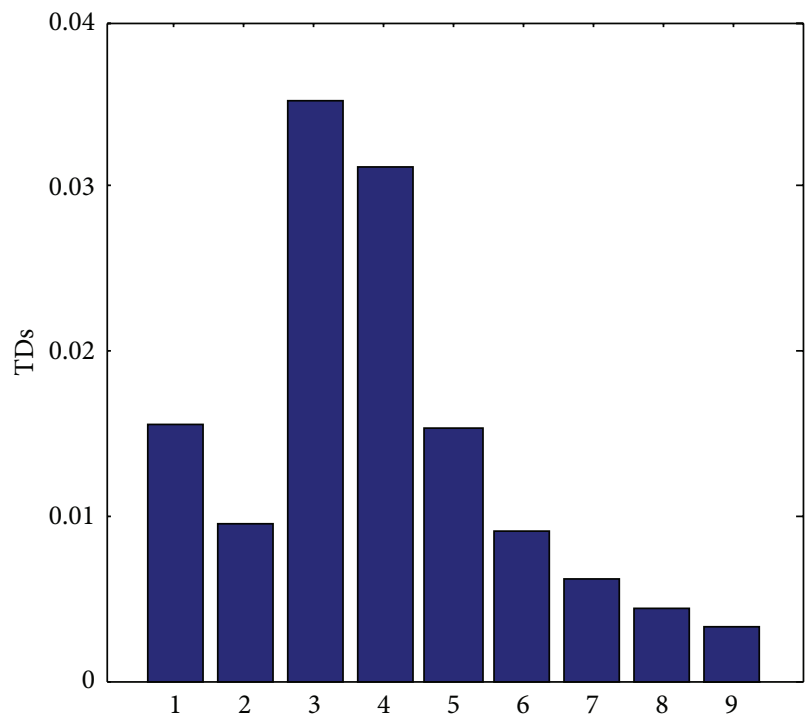

(b)

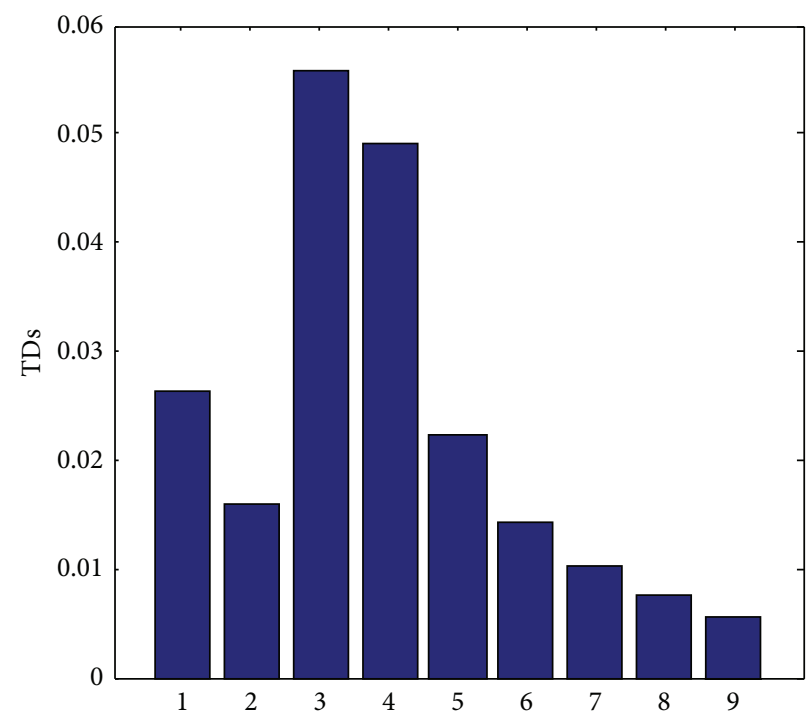

(d)

FIGURE 9: Detection indicators of the cantilever beam for $0-50 \mathrm{~Hz}$ with different crack depth ratios: (a)-(d) are the indicators with $\gamma$ from 0.1 to 0.4 , respectively.

where $\xi_{j}$ is the location of the $j$ th crack within the element and $\xi_{j 1}=\xi_{j}-l_{c}$ and $\xi_{j 2}=\xi_{j}+l_{c}$ are the positions on either side of the crack where the stiffness reduction begins. $I_{0}=w d^{3} / 12$ and $I_{c j}=w\left(d-d_{c j}\right)^{3} / 12$ are the second moment of the areas of the undamaged beam and at the $j$ th crack. $d_{c j}$ is the crack depth of beam with dimensions $L \times w \times d$.
The advantage of this simplified model is that the stiffness reduction is local and the stiffness matrix may be written as an explicit function of the crack location and depth. The location of the crack is set at the center of one element, and $\gamma=d_{c j} / d$ is set as the ratio of crack depth to depth of the beam. Hence, the stiffness matrix is the function of $\gamma$. 


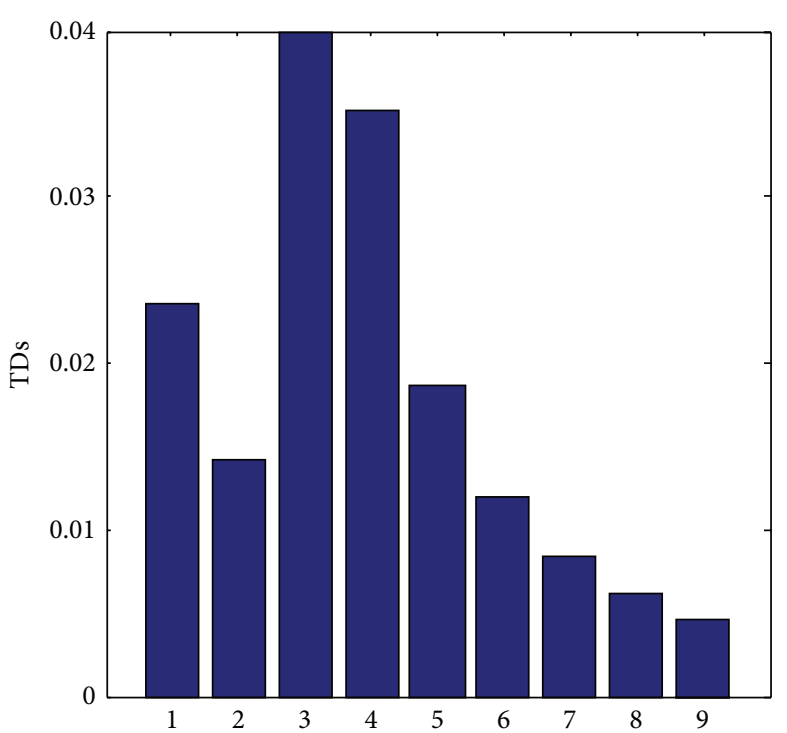

(a)

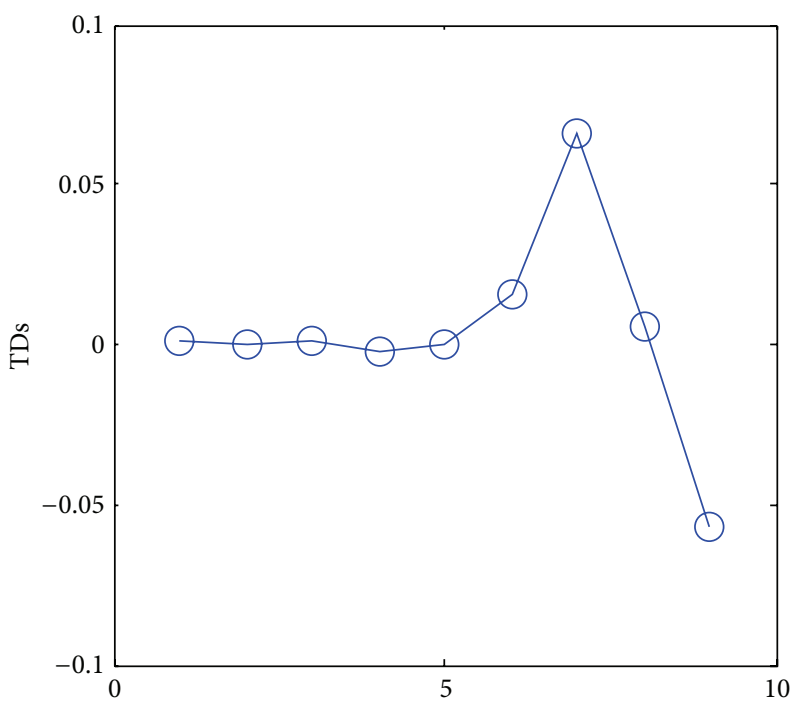

(c)

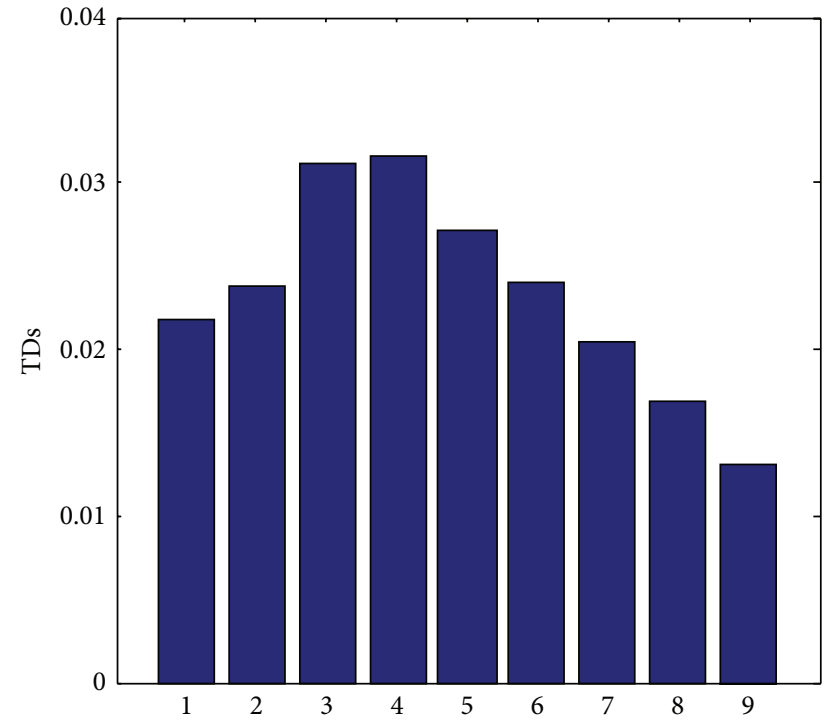

(b)

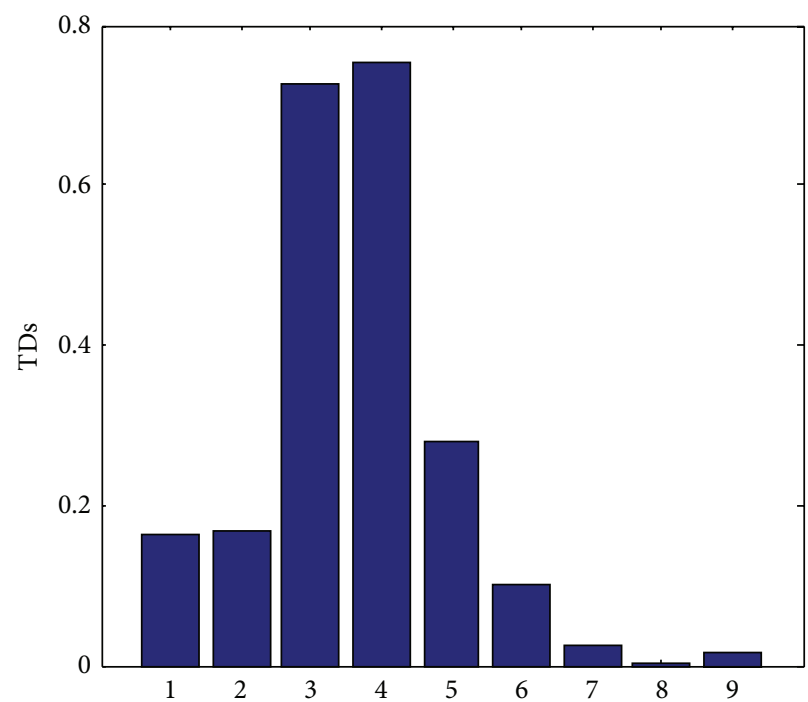

(d)

Figure 10: Detection indicators of the cantilever beam when $\gamma=0.2$ with frequency band of $0-10 \mathrm{~Hz}$ (a) this paper, (b) Schulz et al. [4], (c) magnitude value of Zhang et al. [6], and (d) Johnson and Adams [9].

Figure 8 shows the finite element model of the beam used in this work. The dimensions of the beam are $1.00 \times 0.05 \times$ $0.025 \mathrm{~m}^{3}(L \times w \times d)$. It has a mass density of $2600 \mathrm{~kg} / \mathrm{m}^{3}$, an elastic modulus of $69.79 \mathrm{MPa}$, and a damping ratio of 0.001 . The whole beam is divided into 10 elements. An open crack happens in the 4 th element.

The first three natural frequencies of intact beam and cracked beam are shown in Table 2. Through the comparison, it shows that the natural frequencies change slightly with the increase of crack depth ratio $\gamma$, where the maximum decrease ratio is only $1.699 \%$ for the first natural frequency. That means it is difficult to detect the crack from the natural frequencies directly.
According to the natural frequencies of the beam, a frequency band of $0-50 \mathrm{~Hz}$ is selected for the detection tests in the first, and $20 \mathrm{~N}$ impulse force is applied on the right end of the beam to excite the structure. The fourth-order RungeKutta method is used to calculate the response of the beam. Then, the spectrum can be calculated and TFs can be got.

4.2. Results and Comparison. Figure 9 gives the result of the detection indicators of the cracked cantilever beam in four different damaged levels for frequency band of $0-50 \mathrm{~Hz}$ without noise. Since the responses are measured from the second point of the beam, $\mathrm{TD}_{3}$ represents the indicator of the 4th element, which is the maximum value of all indicators in 
TABLE 2: Comparison of natural frequencies of intact and damaged cantilever beam with different crack depth ratios.

\begin{tabular}{|c|c|c|c|c|c|c|c|c|c|}
\hline \multirow{2}{*}{$\gamma$} & \multicolumn{3}{|c|}{ First mode } & \multicolumn{3}{|c|}{ Second mode } & \multicolumn{3}{|c|}{ Third mode } \\
\hline & Intact & Damaged & Rel. (\%) & Intact & Damaged & Rel. (\%) & Intact & Damaged & Rel. (\%) \\
\hline 0.1 & 4.147 & 4.127 & 0.484 & 11.613 & 11.521 & 0.791 & 22.773 & 22.766 & 0.031 \\
\hline 0.2 & 4.147 & 4.108 & 0.941 & 11.613 & 11.437 & 1.518 & 22.773 & 22.760 & 0.057 \\
\hline 0.3 & 4.147 & 4.091 & 1.352 & 11.613 & 11.363 & 2.153 & 22.773 & 22.756 & 0.078 \\
\hline 0.4 & 4.147 & 4.076 & 1.699 & 11.613 & 11.302 & 2.677 & 22.773 & 22.752 & 0.095 \\
\hline
\end{tabular}

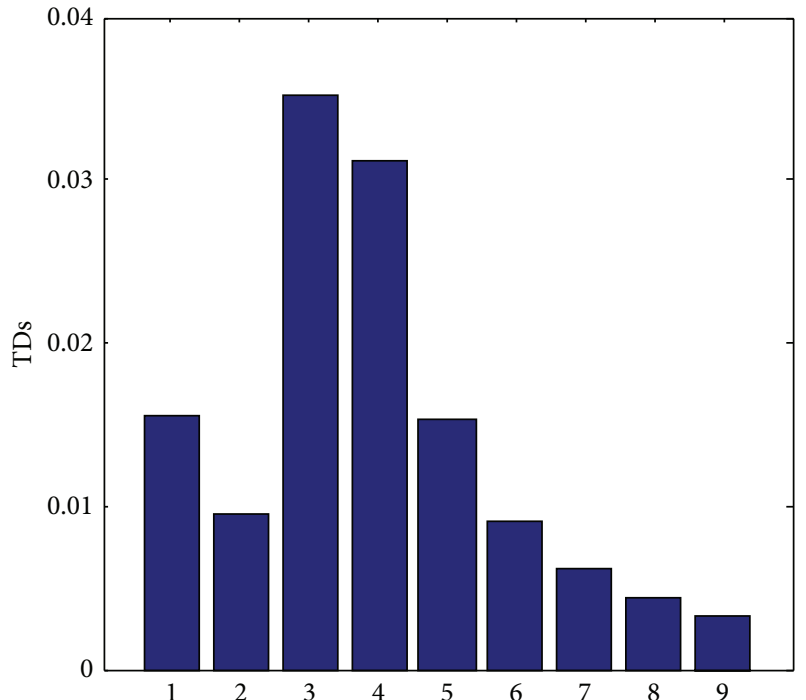

(a)

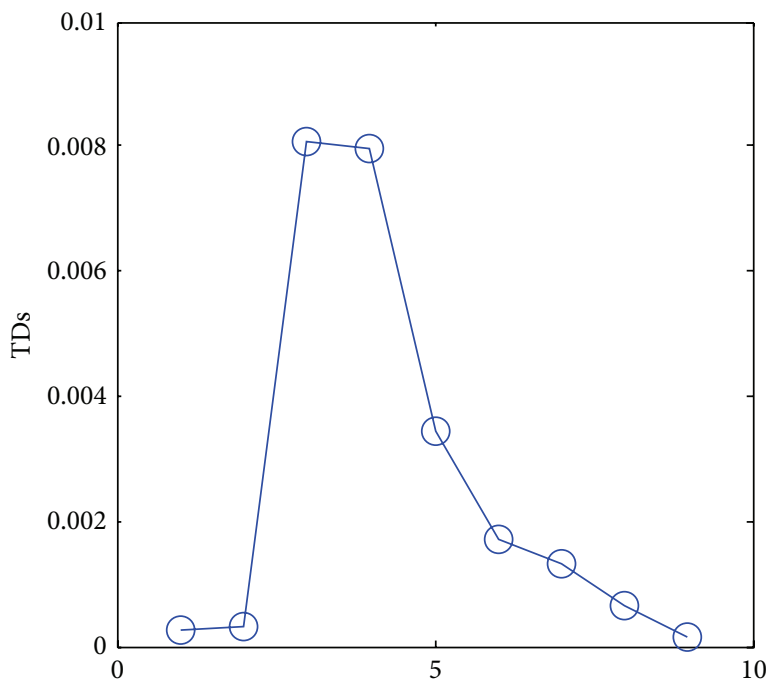

(c)

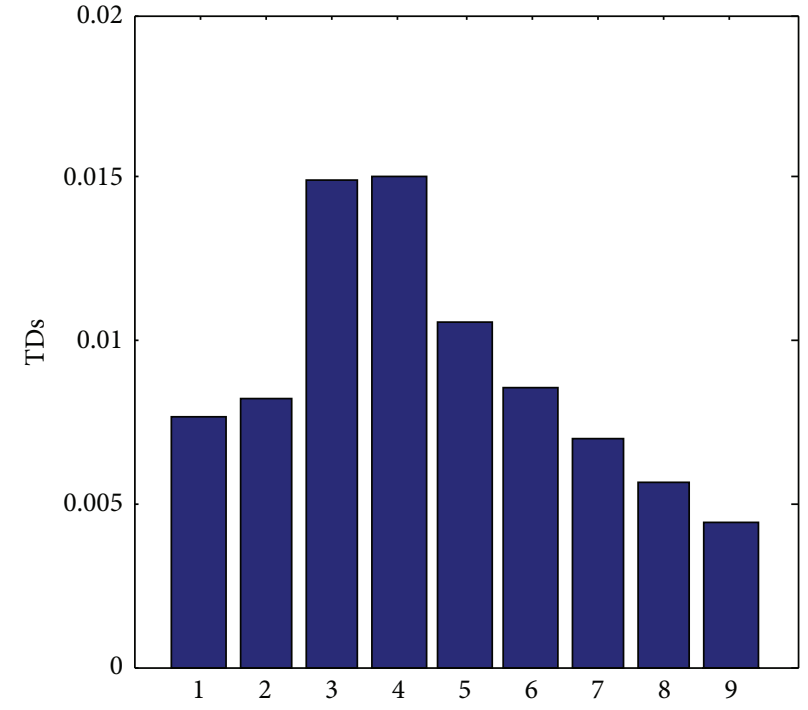

(b)

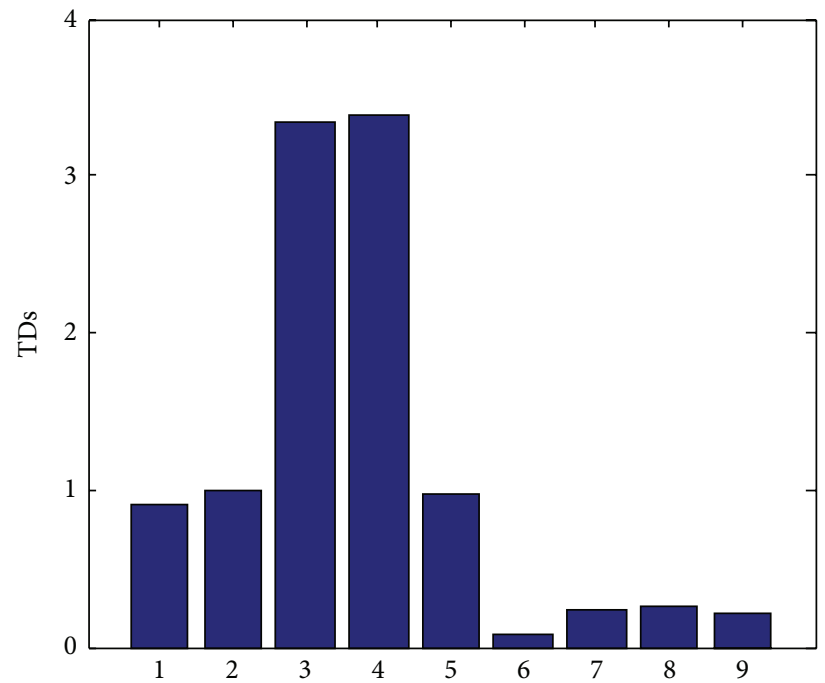

(d)

FIGURE 11: Detection indicators of the cantilever beam when $\gamma=0.2$ with frequency band of 0-50 Hz: (a) this paper, (b) Schulz et al. [4], (c) magnitude value of Zhang et al. [6], and (d) Johnson and Adams [9].

each case. Hence, crack location can be detected and located correctly, while, for $\gamma$ equal to 0.1 and $0.3, \mathrm{TD}_{1}$ is very big, which means that the detection indicators are influenced by the boundary conditions.

Since the damage indicators based on transmissibility functions are sensitive to the frequency bands and noise [5], a comparison will be made to study the advantages of the proposed method in this paper. The indicator proposed in this paper is compared with the indicators defined by Schulz et al. [4], Zhang et al. [6], and Johnson and Adams [9] in the same damage conditions with different frequency bands or different noise levels. 


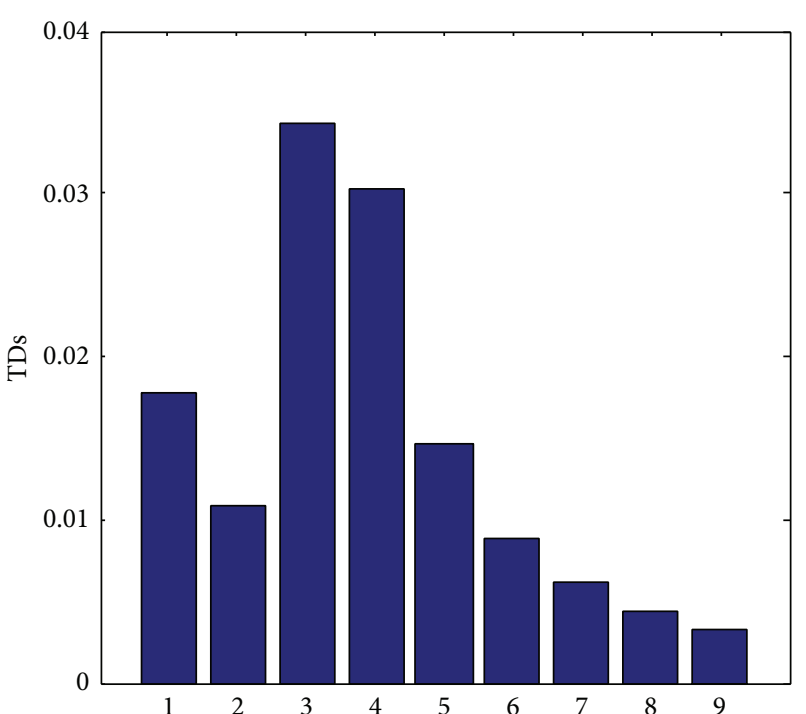

(a)

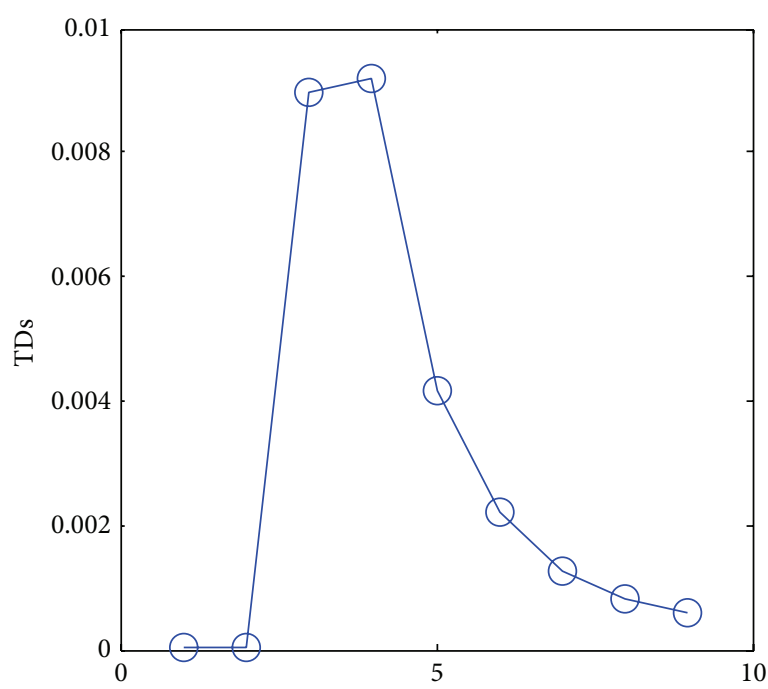

(c)

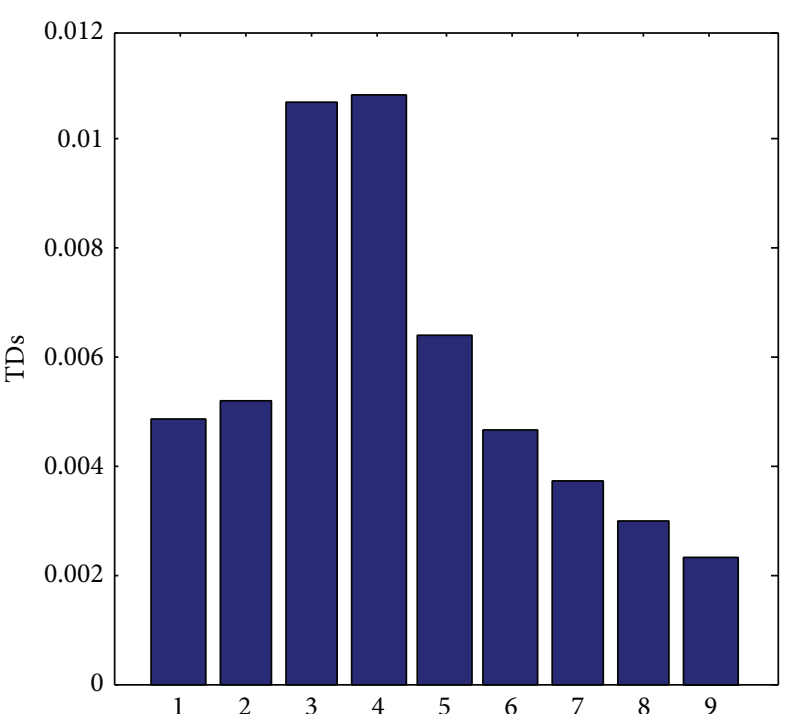

(b)

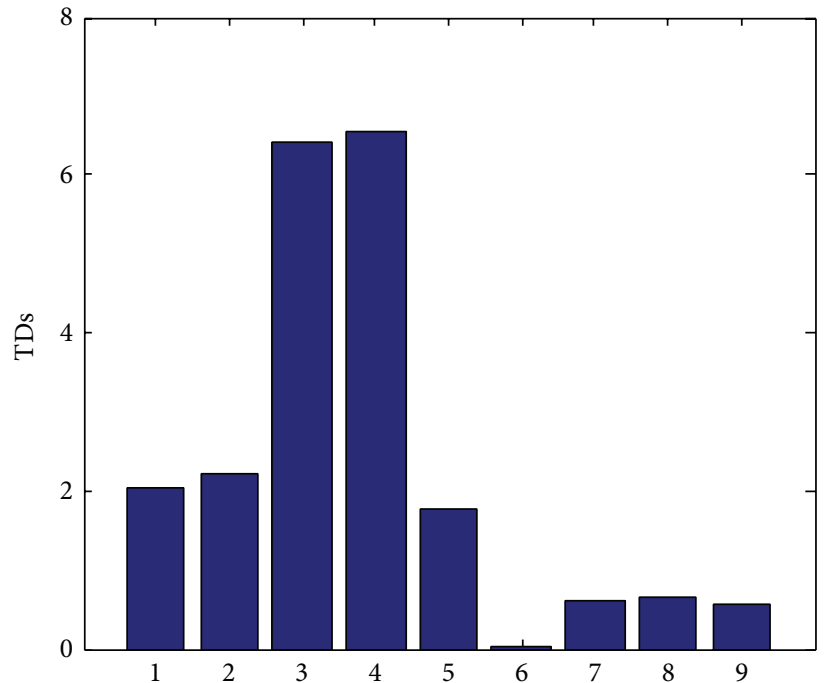

(d)

FIGURE 12: Detection indicators of the cantilever beam when $\gamma=0.2$ with frequency band of 0-100 Hz: (a) this paper, (b) Schulz et al. [4], (c) magnitude value of Zhang et al. [6], and (d) Johnson and Adams [9].

4.2.1. Effect of Frequency Bands. The effect of frequency bands is studied in this application. Considering the natural frequency in Table 2, and three different frequency bands, $0-10 \mathrm{~Hz}, 0-50 \mathrm{~Hz}$, and $0-100 \mathrm{~Hz}$, are used to compute the indicators. The crack depth ratio $\gamma$ is 0.2 , and stiffness reduction of the beam element is no more than $8.5 \%$.

Figure 10 shows the indicators of the cantilever beam for frequency band of $0-10 \mathrm{~Hz}$. It can be seen that the method proposed by this paper locates the crack position correctly. For indicators proposed by Zhang et al. [6], the magnitude value gives the complete wrong location. While the conventional method by Schulz et al. [4] and quotients of transmissibility ratios by Johnson and Adams [9] also misdetect the crack for this frequency band, the indicator of the fourth element is maximum, which is near the crack position.
Figures 11 and 12 show the detection indicators for frequency bands of $0-50 \mathrm{~Hz}$ and $0-100 \mathrm{~Hz}$, separately. Besides the indicators proposed by this paper, the other three methods still cannot locate the crack all the time. But all these indicators give the crack position very close to the right location. For all these three frequency bands, the method proposed by this paper locates the crack correctly, which means that this method is more robust to the frequency band compared to the other three methods.

4.2.2. Effect of Noise. Besides the frequency band, measurement inaccuracy is another influence factor to the transmissibility based detection indicators. In this section, Gaussian noise is added on response directly, and the sensitivity to noise of the method is studied. Once again, scenario with 


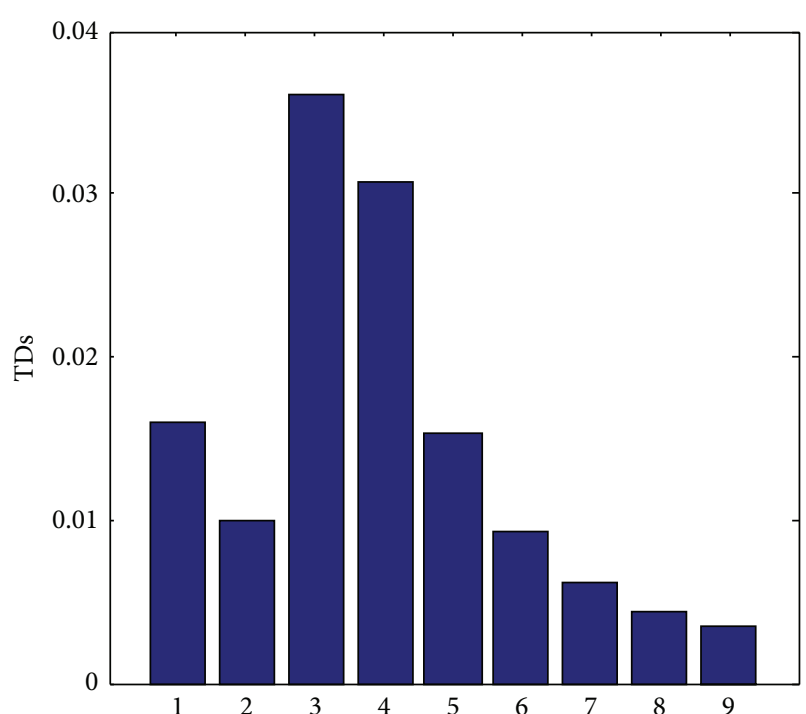

(a)

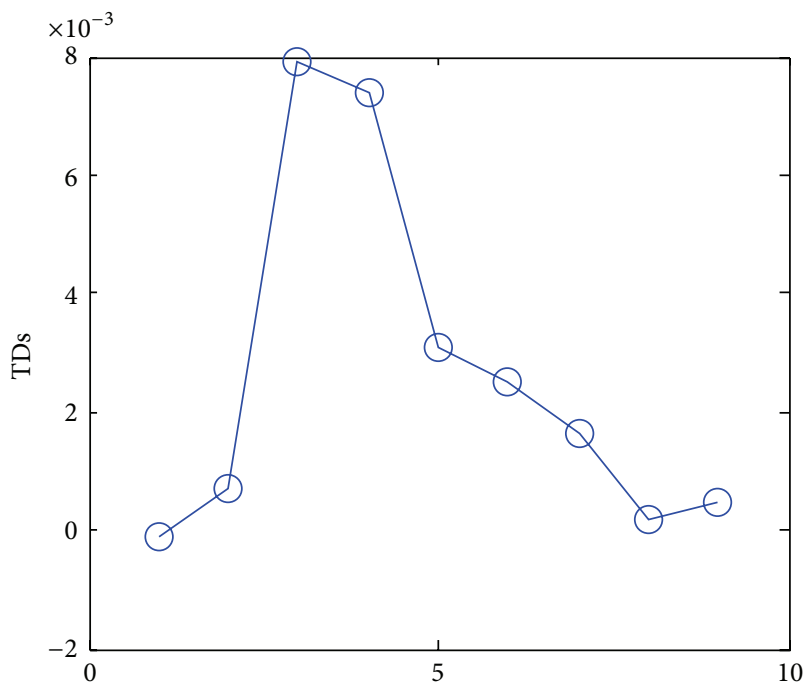

(c)

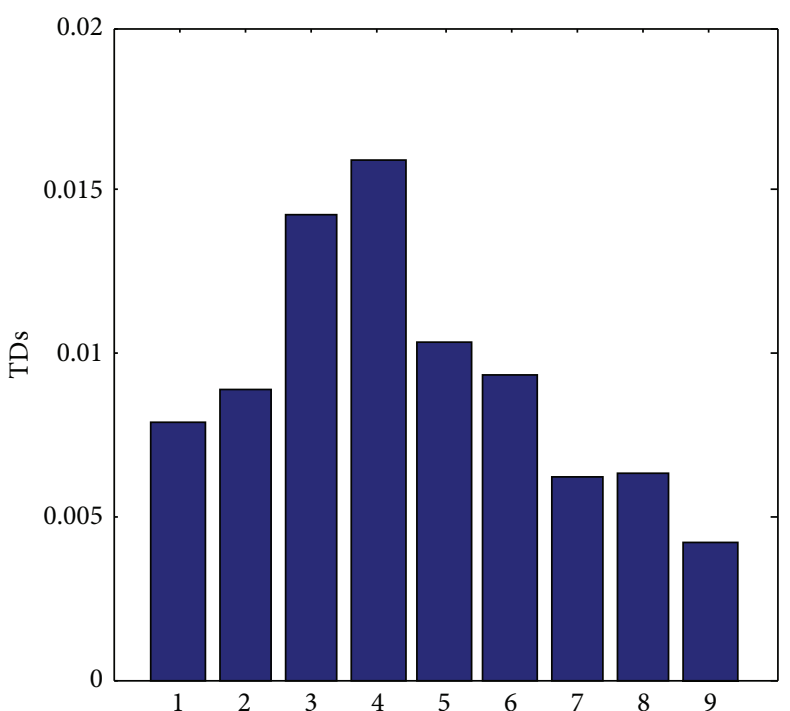

(b)

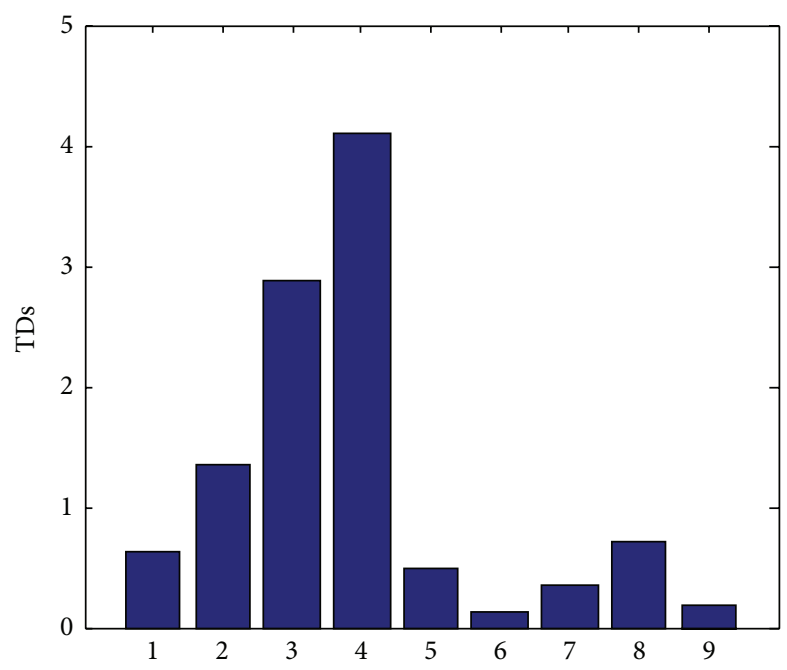

(d)

FIGURE 13: Detection indicators of the cantilever beam when $\gamma=0.2$ for frequency band of $0-50 \mathrm{~Hz}$ with $60 \mathrm{~dB}$ : (a) this paper, (b) Schulz et al. [4], (c) magnitude value of Zhang et al. [6], and (d) Johnson and Adams [9].

crack depth ratio of 0.2 is selected. According to the study of frequency band, $0-50 \mathrm{~Hz}$ is set as the suitable frequency band.

Three levels of noise, $60 \mathrm{~dB}, 40 \mathrm{~dB}$, and $20 \mathrm{~dB}$, are taken into consideration. When the noise is low, detection indicators change slightly in Figure 13 except for method proposed by Johnson and Adams [9]. The indicators of methods proposed by this paper and Zhang et al. [6] all give the right crack position, and the conventional method proposed by Schulz et al. [4] locates the fourth element, which is close to the crack. And when the noise grows in Figures 14 and 15, only the indicators of methods proposed by this paper and Zhang et al. [6] can give the right position or position near the crack. The indicators by Schulz et al. [4] and Johnson and Adams [9] are completely disordered, and the crack is totally misdirected. Even when the noise is $20 \mathrm{~dB}$, the method proposed by this paper still can locate the crack correctly. Therefore, the proposed method in this paper can decrease the influence of noise using weight function compared with the other three methods.

\section{Conclusions}

In this paper, the effects of the local variation on the TFs are studied, and the results show that TFs between the position of local variation and input change obviously, while the others 


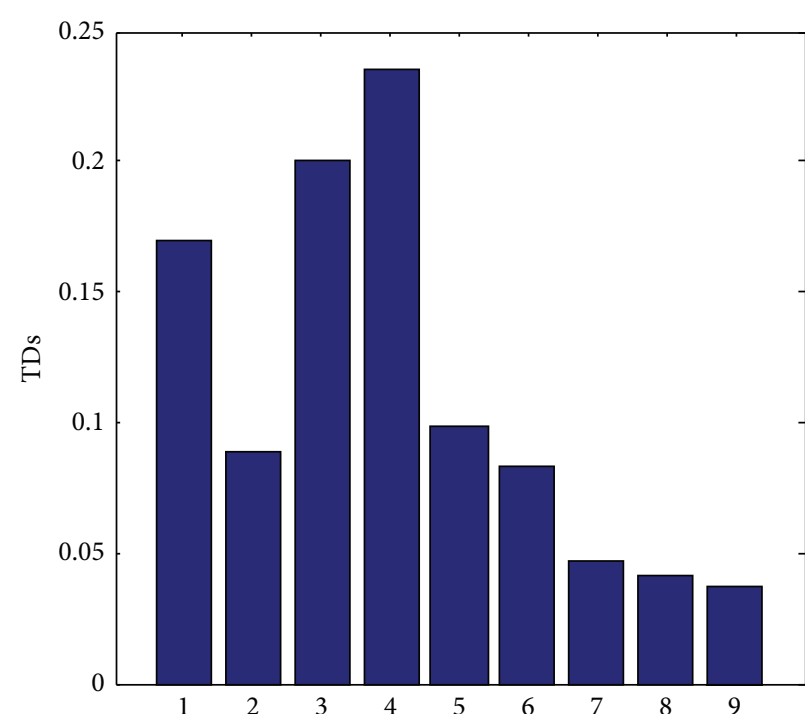

(a)

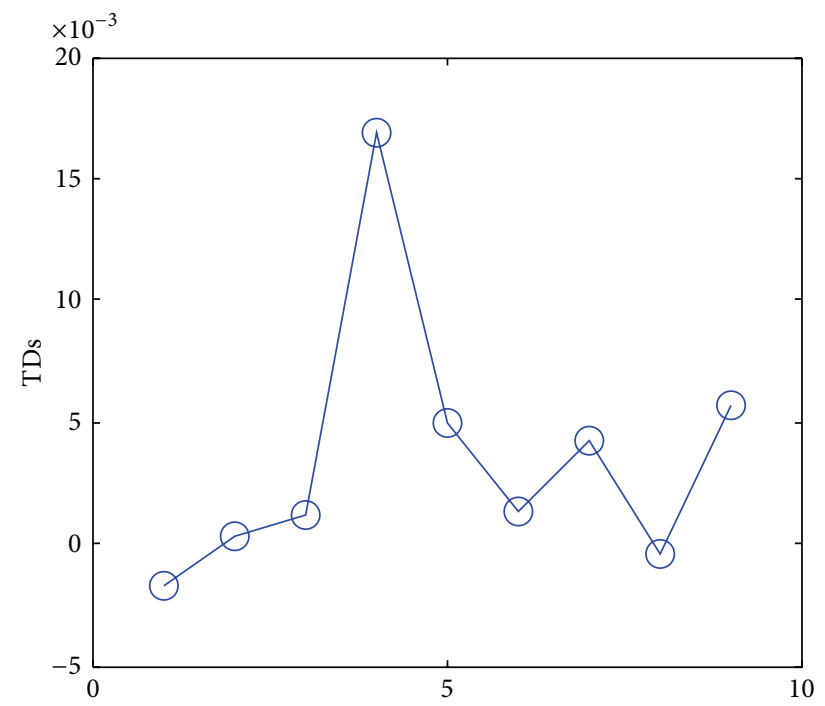

(c)

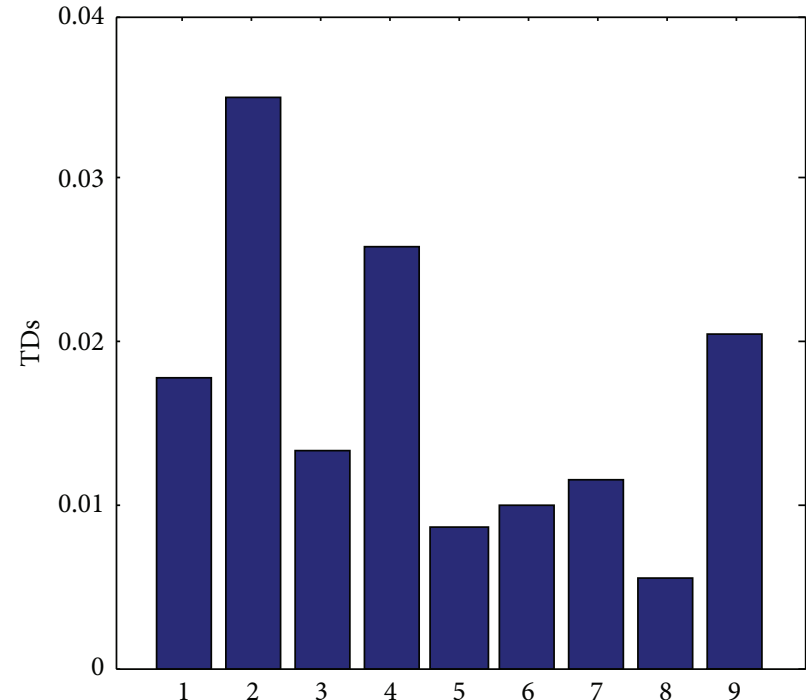

(b)

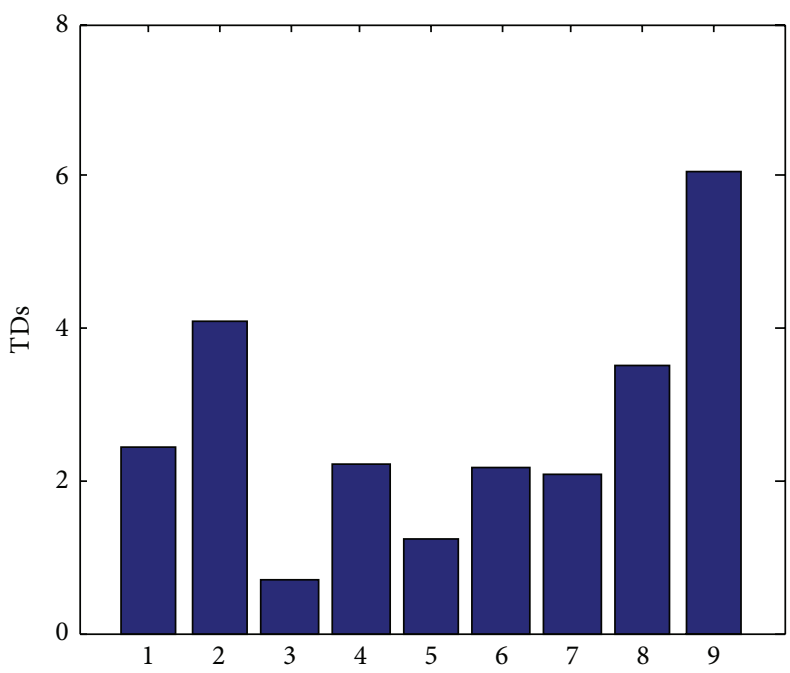

(d)

Figure 14: Detection indicators of the cantilever beam when $\gamma=0.2$ for frequency band of $0-50 \mathrm{~Hz}$ with $40 \mathrm{~dB}$ : (a) this paper, (b) Schulz et al. [4], (c) magnitude value of Zhang et al. [6], and (d) Johnson and Adams [9].

remain constant. Based on these properties, the differences between the transmissibility functions before and after the variation are used to develop a new detection indicator.

Through the simulation of linear, bilinear, and nonlinear one-dimensional MDOF system, the indicator was validated. And the detection indicators were applied in the crack detection in beams structures. From the study of the properties and the application of TFs, it was found that TFs are influenced by many factors, such as the local restoring force, location of external force, boundary, and frequency band. Even for a defined system, it is not easy to determine proper frequency bands to avoid the influence of the subordinate factors. But this paper indeed shows the advantage of the new indicator in local variation detection through the comparison with the other three indicators based on transmissibility functions. The new indicator is more robust to the frequency bands and noise.

Based on the study above, the authors will focus on reducing the influence of these factors on the transmissibility in one-dimensional and multidimensional MDOF system in the future.

\section{Conflict of Interests}

The authors declare that there is no conflict of interests regarding the publication of this paper. 


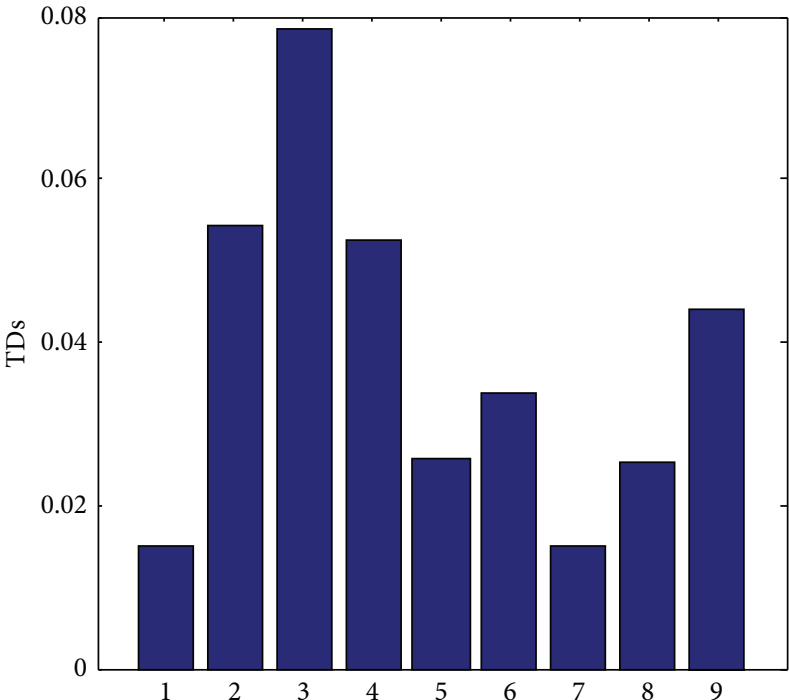

(a)

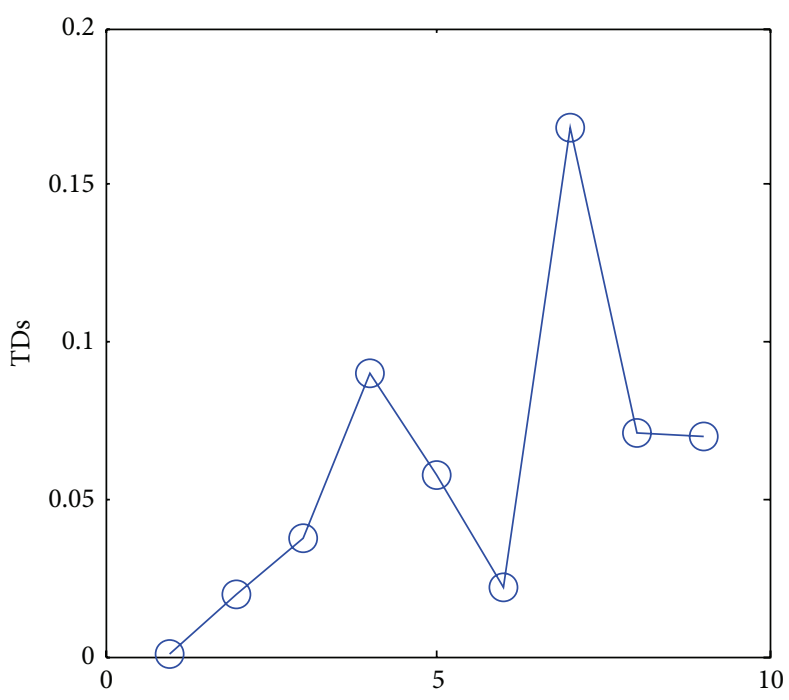

(c)

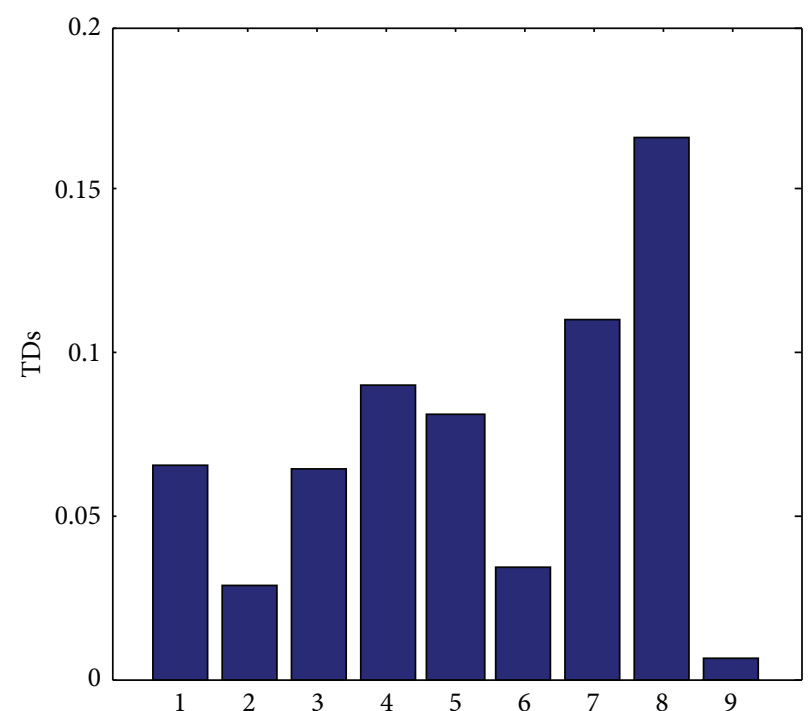

(b)

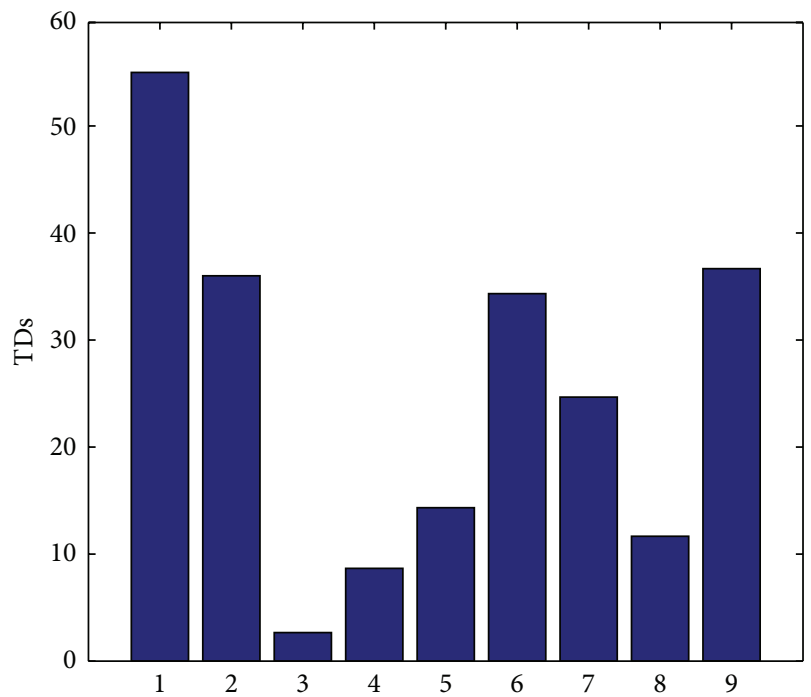

(d)

FIGURE 15: Detection indicators of the cantilever beam when $\gamma=0.2$ for frequency band of 0-50 Hz with $20 \mathrm{~dB}$ : (a) this paper, (b) Schulz et al. [4], (c) magnitude value of Zhang et al. [6], and (d) Johnson and Adams [9].

\section{Acknowledgment}

The authors gratefully acknowledge that this paper was supported by the NSFC for Distinguished Young Scholars (11125209) and the NSFC (11472170, 51421092).

\section{References}

[1] W. Fan and P. Qiao, "Vibration-based damage identification methods: a review and comparative study," Structural Health Monitoring, vol. 10, no. 1, pp. 83-111, 2011.

[2] C. C. Ciang, J.-R. Lee, and H.-J. Bang, "Structural health monitoring for a wind turbine system: a review of damage detection methods," Measurement Science and Technology, vol. 19, no. 12, Article ID 122001, 2008.
[3] Z. Q. Su, L. Ye, and Y. Lu, "Guided Lamb waves for identification of damage in composite structures: a review," Journal of Sound and Vibration, vol. 295, no. 3-5, pp. 753-780, 2006.

[4] M. J. Schulz, A. S. Naser, P. F. Pai, M. S. Linville, and J. Chung, "Detecting structural damage using transmittance functions," in Proceedings of the 15th International Modal Analysis Conference (IMAC '97), pp. 638-644, 1997.

[5] S. Chesné and A. Deraemaeker, "Damage localization using transmissibility functions: a critical review," Mechanical Systems and Signal Processing, vol. 38, no. 2, pp. 569-584, 2013.

[6] H. Zhang, M. J. Schulz, A. Naser, F. Ferguson, and P. F. Pai, "Structural health monitoring using transmittance functions," Mechanical Systems and Signal Processing, vol. 13, no. 5, pp. 765$787,1999$. 
[7] J. E. Mottershead, "On the zeros of structural frequency response functions and their sensitivities," Mechanical Systems and Signal Processing, vol. 12, no. 5, pp. 591-597, 1998.

[8] Q. Chen, Y. W. Chan, and K. Worden, "Structural fault detection using neural networks trained on transmissibility functions," in Proceedings of the International Conference on Vibration Engineering, pp. 446-456, Beijing, China, 1994.

[9] T. J. Johnson and D. E. Adams, "Transmissibility as a differential indicator of structural damage," Journal of Vibration \& Acoustics, vol. 124, no. 4, pp. 634-641, 2002.

[10] C. Devriendt and P. Guillaume, "Identification of modal parameters from transmissibility measurements," Journal of Sound and Vibration, vol. 314, no. 1-2, pp. 343-356, 2008.

[11] W.-J. Yan and W.-X. Ren, "Operational modal parameter identification from power spectrum density transmissibility," Computer-Aided Civil \& Infrastructure Engineering, vol. 27, no. 3, pp. 202-217, 2012.

[12] N. M. M. Maia, R. A. B. Almeida, A. P. V. Urgueira, and R. P. C. Sampaio, "Damage detection and quantification using transmissibility," Mechanical Systems and Signal Processing, vol. 25, no. 7, pp. 2475-2483, 2011.

[13] J. Li and H. Hao, "Damage detection of shear connectors based on power spectral density transmissibility," Key Engineering Materials, vol. 569-570, pp. 1241-1248, 2013.

[14] J. Li, H. Hao, Y. Xia, and H.-P. Zhu, "Damage detection of shear connectors in bridge structures with transmissibility in frequency domain," International Journal of Structural Stability \& Dynamics, vol. 14, no. 2, Article ID 1350061, pp. 634-640, 2014.

[15] J. Li, H. Hao, and J. V. Lo, "Structural damage identification with power spectral density transmissibility: numerical and experimental studies," Smart Structures \& Systems, vol. 15, no. 1, pp. 15-40, 2015.

[16] Y. L. Zhou, E. Figueiredo, N. Maia, and R. Perera, "Damage detection and quantification using transmissibility coherence analysis," Shock and Vibration, vol. 2015, Article ID 290714, 16 pages, 2015.

[17] Y. L. Zhou, E. Figueiredo, N. Maia, R. Sampaio, and R. Perera, "Damage detection in structures using a transmissibility-based Mahalanobis distance," Structural Control \& Health Monitoring, 2015.

[18] H. Jeffreys and B. Swirles, Methods of Mathematical Physics, Cambridge University Press, Cambridge, UK, 1999.

[19] J. L. Schiff, The laplace Transform: Theory and Applications, Springer, New York, NY, USA, 1999.

[20] P. Cawley and R. D. Adams, "The location of defects in structures from measurements of natural frequencies," Journal of Strain Analysis for Engineering Design, vol. 14, no. 2, pp. 49-57, 1979.

[21] A. K. Pandey and M. Biswas, "Damage detection in structures using changes in flexibility," Journal of Sound \& Vibration, vol. 169, no. 1, pp. 3-17, 1994.

[22] A. Rivola and P. R. White, "Bispectral analysis of the bilinear oscillator with application to the detection of fatigue cracks," Journal of Sound \& Vibration, vol. 216, no. 5, pp. 889-910, 1998.

[23] J. N. Sundermeyer and R. L. Weaver, "On crack identification and characterization in a beam by non-linear vibration analysis," Journal of Sound and Vibration, vol. 183, no. 5, pp. 857-871, 1995.

[24] Y. C. Chu and M.-H. H. Shen, "Analysis of forced bilinear oscillators and the application to cracked beam dynamics," AIAA Journal, vol. 30, no. 10, pp. 2512-2519, 1992.
[25] S. V. Walker, J.-Y. Kim, J. Qu, and L. J. Jacobs, "Fatigue damage evaluation in A36 steel using nonlinear Rayleigh surface waves," NDT \& E International, vol. 48, pp. 10-15, 2012.

[26] C. Bermes, J.-Y. Kim, J. Qu, and L. J. Jacobs, "Experimental characterization of material nonlinearity using lamb waves," Applied Physics Letters, vol. 90, no. 2, Article ID 021901, 3 pages, 2007.

[27] S. S. Kessler, S. M. Spearing, and C. Soutis, "Damage detection in composite materials using Lamb wave methods," Smart Materials \& Structures, vol. 11, no. 2, pp. 269-278, 2002.

[28] W. Li, Y. Cho, T. Ju, H. Choi, N. Kim, and I. Park, "Evaluation of material degradation of composite laminates using nonlinear lamb wave," in Nondestructive Testing of Materials and Structures, pp. 593-598, Springer, Amsterdam, The Netherlands, 2013.

[29] M. I. Friswell and J. E. T. Penny, "Crack modeling for structural health monitoring," Structural Health Monitoring, vol. 1, no. 2, pp. 139-148, 2002.

[30] A. Khorram, M. Rezaeian, and F. Bakhtiari-Nejad, "Multiple cracks detection in a beam subjected to a moving load using wavelet analysis combined with factorial design," European Journal of Mechanics-A/Solids, vol. 40, no. 1, pp. 97-113, 2013.

[31] G. M. Owolabi, A. S. J. Swamidas, and R. Seshadri, "Crack detection in beams using changes in frequencies and amplitudes of frequency response functions," Journal of Sound \& Vibration, vol. 265, no. 1, pp. 1-22, 2003.

[32] A. O. Ayhan, "Simulation of three-dimensional fatigue crack propagation using enriched finite elements," Computers \& Structures, vol. 89, no. 9-10, pp. 801-812, 2011.

[33] M. Götting and J. Rösler, "A finite element creep crack growth model for waspaloy," Computers \& Structures, vol. 85, no. 3-4, pp. 225-232, 2007.

[34] J. K. Sinha, M. I. Friswell, and S. Edwards, "Simplified models for the location of cracks in beam structures using measured vibration data," Journal of Sou \& Vibration, vol. 251, no. 1, pp. 13-38, 2002. 

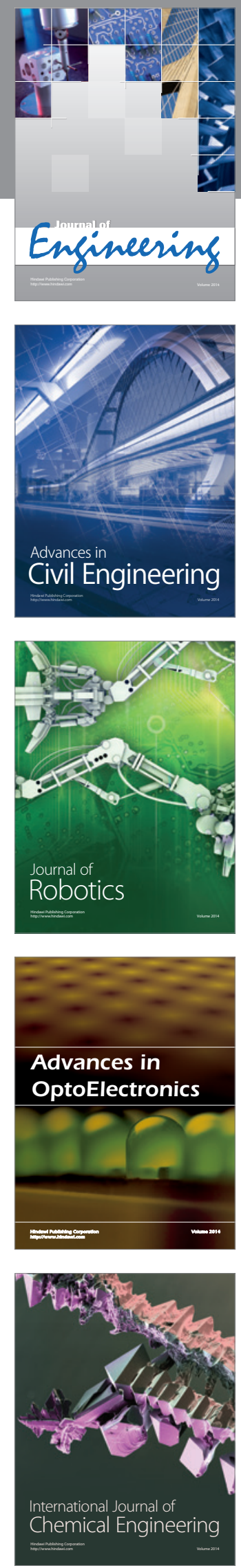

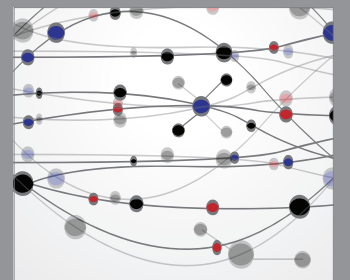

The Scientific World Journal
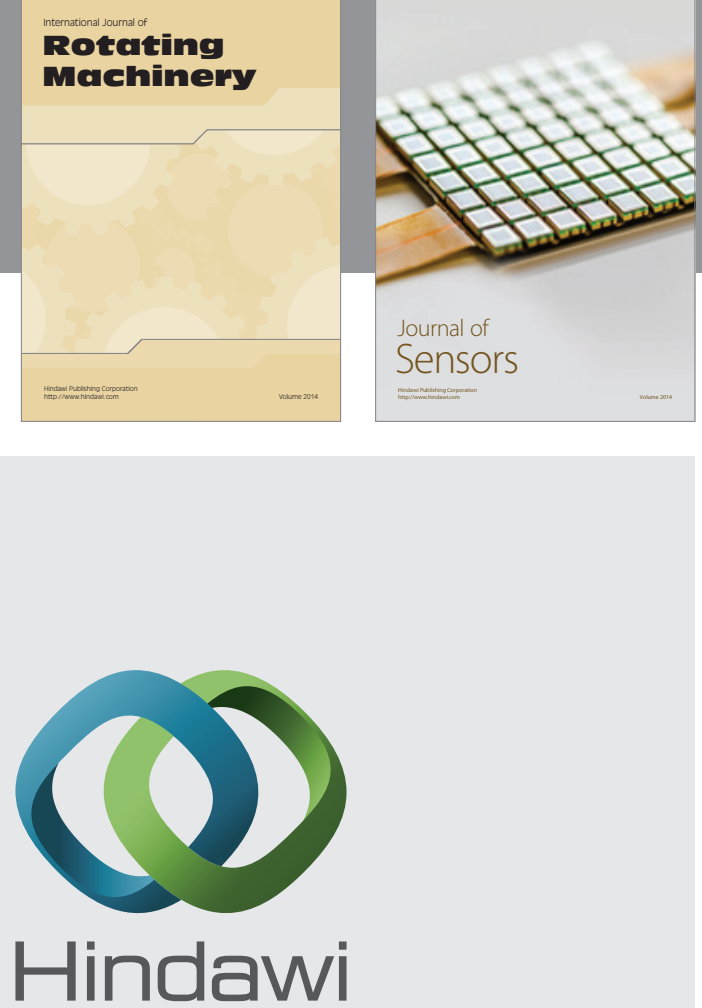

Submit your manuscripts at http://www.hindawi.com
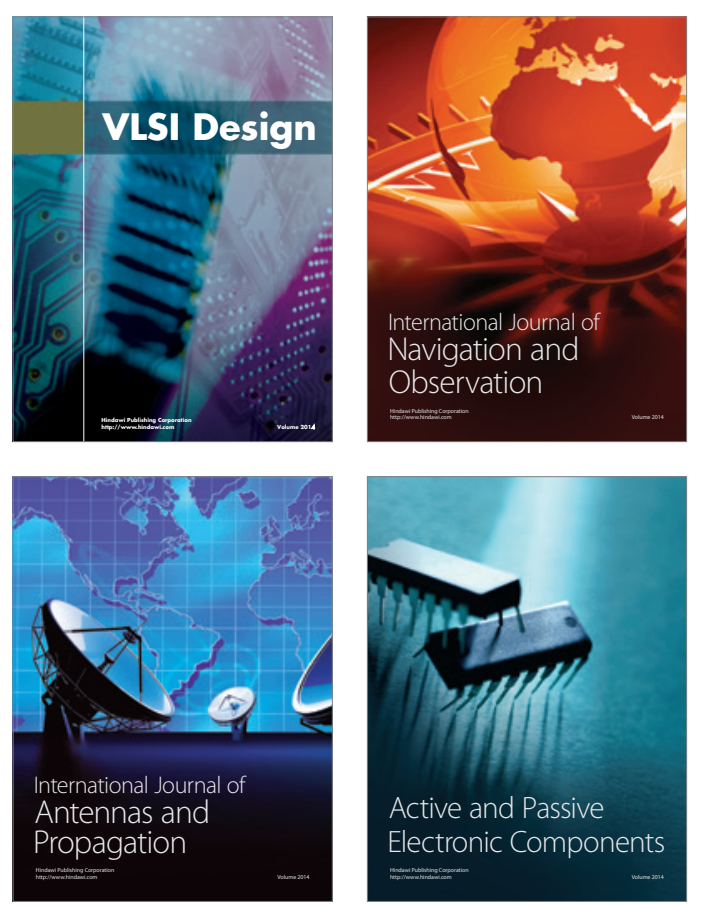
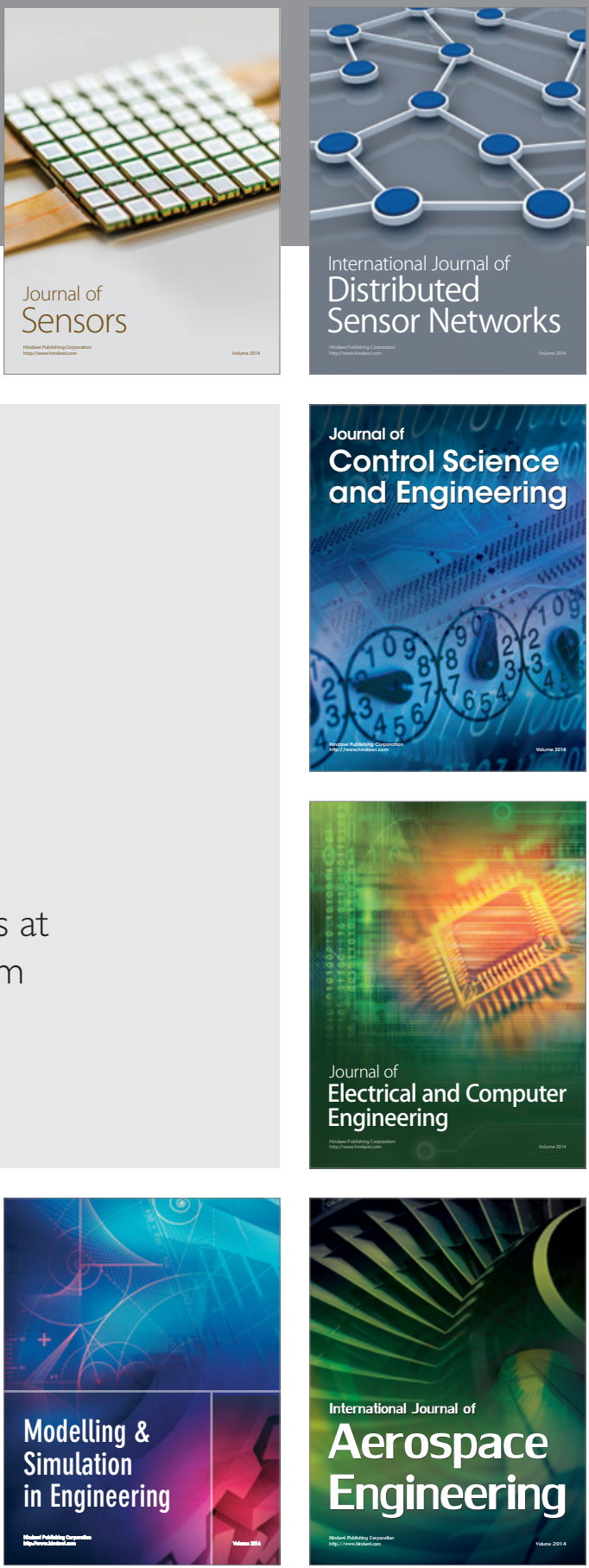

Journal of

Control Science

and Engineering
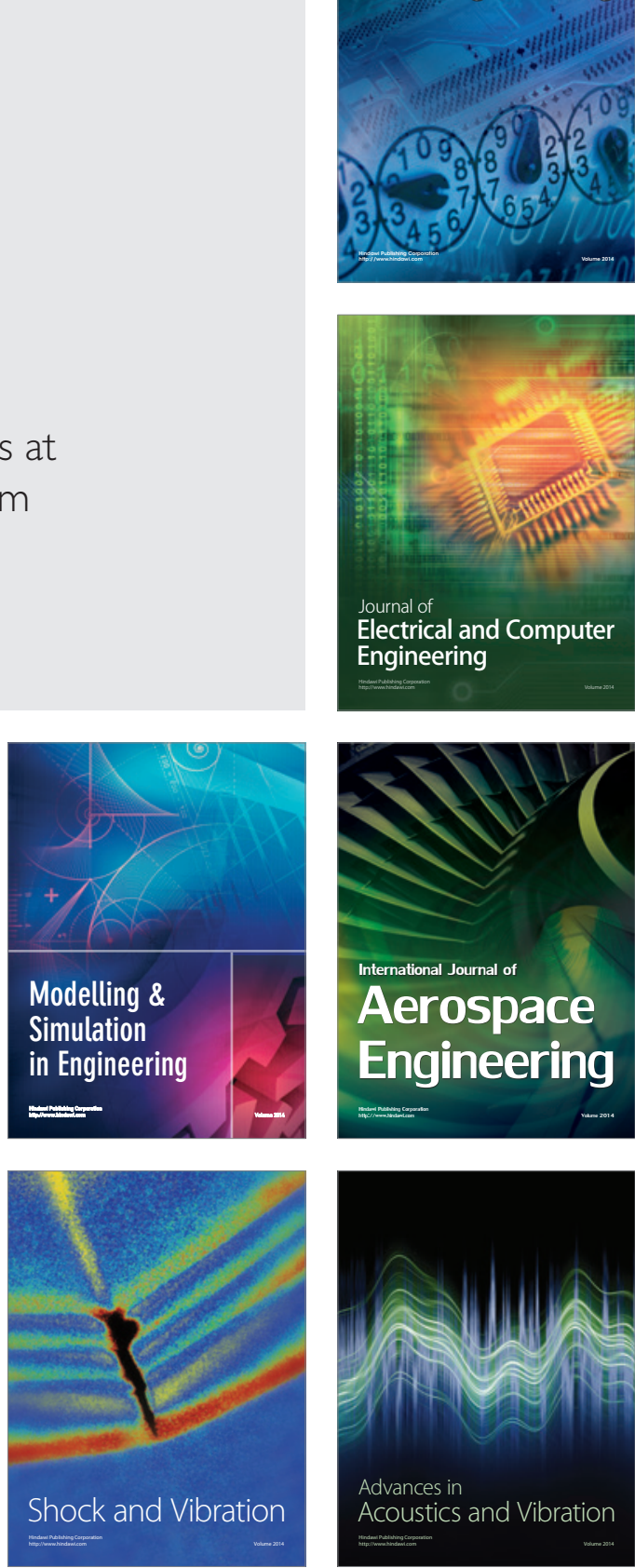